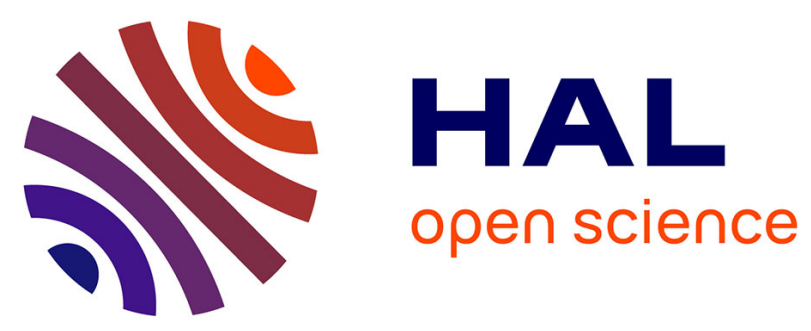

\title{
Evaluation d un logiciel éducatif dans deux contextes de communication : face à face et synchrone à distance
}

\author{
Aurélie Lainé, Alexandra Wallner, Annick Weil-Barais
}

\section{To cite this version:}

Aurélie Lainé, Alexandra Wallner, Annick Weil-Barais. Evaluation d un logiciel éducatif dans deux contextes de communication : face à face et synchrone à distance. STICEF (Sciences et Technologies de l'Information et de la Communication pour l'Éducation et la Formation), 2006, 13, 27 p. hal00696333

\section{HAL Id: hal-00696333 \\ https://hal.science/hal-00696333}

Submitted on 11 May 2012

HAL is a multi-disciplinary open access archive for the deposit and dissemination of scientific research documents, whether they are published or not. The documents may come from teaching and research institutions in France or abroad, or from public or private research centers.
L'archive ouverte pluridisciplinaire HAL, est destinée au dépôt et à la diffusion de documents scientifiques de niveau recherche, publiés ou non, émanant des établissements d'enseignement et de recherche français ou étrangers, des laboratoires publics ou privés. 


\title{
Evaluation d'un logiciel éducatif dans deux contextes de communication : face à face et synchrone à distance
}

\author{
Aurélie LAINÉ, Alexandra WALLNER, Annick WEIL-BARAIS \\ [Université d'Angers]
}

\begin{abstract}
- RÉSUMÉ : La recherche présentée dans cet article compare les échanges entre pairs dans différentes situations de collaboration (en face à face ou à distance) impliquant soit des collégiens, soit des étudiants d'université confrontés à une tâche de construction d'une carte conceptuelle rendant compte du réseau conceptuel exprimé dans un texte explicatif, à l'aide du logiciel ModellingSpace. La méthodologie des deux études décrites s'appuie sur un examen de la littérature scientifique concernant les facteurs ayant une incidence sur les interactions. La première étude a impliqué 11 dyades d'étudiants universitaires en 3ème année d'étude de psychologie ; la moitié des dyades communique en face à face, et l'autre moitié à distance. Dans la seconde étude, la modalité de communication à distance a été répliquée auprès d'élèves d'une classe de 4ème (âgés de 14 ans en moyenne), 8 dyades au total. Les analyses concernent la structure et le contenu des échanges. Il s'avère que, chez les étudiants, le mode de communication a un impact sur les échanges enregistrés du point de vue de la structure des échanges, mais pas de leur contenu. Cette différence n'a pas d'incidence sur la qualité des productions. Les échanges des adultes et des adolescents communiquant à distance présentent le même type de structure mais les contenus des échanges sont différents. Ces résultats font l'objet d'une discussion en relation avec des préoccupations liées à l'évaluation des logiciels éducatifs.
\end{abstract}

MOTS CLÉS : Collaboration, communication synchrone à distance, communication en face à face, carte conceptuelle, élèves de collège, étudiants.

- ABSTRACT : In this paper, we compare peer interactions in two collaborative contexts (synchronous textual communication and face-to-face communication) involving either 14-year-old students or university students, who had to build a concept map with an educational software (ModellingSpace). The research methodology of the two studies reported is based on the factors influencing peer interactions in educational context. The first study involved 22 pairs of university student (3rd year of study in psychology); half of them communicated face-to-face and the other group used computer-supported communication. The distant communication condition has been experimented in the second study, with 16 pairs of secondary school students. The structure and content of verbal interactions were the focus of analysis. Results show that the mode of communication has an impact on the structure of the interactions, but not on the content. This difference does not affect the quality of the concept maps. The adults and teenagers' exchanges present the same structure but differ in their content. These results are discussed in relation with the issue of the assessment of educational software.

KEYWORDS : Collaboration, synchronous textual communication, face-to-face communication, concept map, students 


\author{
1 Introduction \\ 2 Le contexte de collaboration \\ 3 Présentation des études \\ 4 Discussion \\ 5 Références
}

\title{
1 Introduction
}

De plus en plus de recherches sur l'enseignement et l'apprentissage adoptent une perspective où l'apprentissage est vu comme un processus social plutôt qu'individuel (Palincsar, 1998) ; (Weil-Barais, 2004) à la suite de très nombreuses études ayant mis en évidence l'importance des interactions sociales, entre l'enseignant et les élèves, mais aussi entre pairs. L'utilisation de plus en plus répandue des technologies de l'information et de la communication en éducation permet la création d'environnements favorisant les interactions. Dans de tels environnements, les élèves peuvent travailler conjointement et communiquer en face à face ou à distance (Jehng, 1997), si le logiciel intègre des fonctions de communication. Ces nouvelles situations de communication médiatisée présentent des caractéristiques susceptibles d'influer sur les interactions des élèves. La recherche présentée ici poursuit comme objectif d'évaluer l'impact de différentes formes de communication entre pairs dans différentes situations de collaboration médiatisée par ordinateur (en face à face ou à distance) impliquant soit des collégiens, soit des étudiants d'université. On connaît mal en effet l'incidence des contraintes qu'imposent les formes de communication à distance sur la qualité des échanges, à différents âges. Les études présentées concernent des élèves de collège et des étudiants d'université qui se différencient notamment par des différences quant aux pratiques d'écriture. On peut faire l'hypothèse que la communication écrite mieux maîtrisée chez les étudiants universitaires affectera moins la qualité et le contenu des échanges que chez les collégiens.

Dans un premier temps, nous rappellerons quelques études fondamentales concernant la collaboration entre pairs qui nous ont permis de dégager les critères d'évaluation pris en compte. Dans un second temps, nous présenterons l'environnement informatique utilisé permettant une communication synchrone et à distance ainsi que la tâche réalisée par les élèves et les étudiants à l'aide de cet environnement. Dans un troisième temps, nous présenterons les études conduites aux plans de la méthodologie employée et des données obtenues. Enfin, les résultats seront discutés dans la perspective de dégager l'intérêt et les limites de telles études pour l'évaluation des logiciels.

\subsection{Collaboration et interactions entre pairs}

L'apprentissage collaboratif est un mode d'apprentissage où l'étudiant apprend grâce aux interactions avec ses pairs (Johnson et Johnson, 1998). De nombreuses études ont montré qu'on apprenait davantage en collaborant avec ses pairs qu'en travaillant de manière isolée. En effet, un environnement d'apprentissage collaboratif accroît la communication et apporte bien plus qu'un simple partage d'idées (Pugach et Johnson, 1995).

La supériorité de l'apprentissage co-actif, coopérant ou en situation de collaboration ou co-résolution a été mise en évidence dans des situations de résolution de problèmes, propices aux échanges interindividuels dans des cas de collaboration "en face à face" (Gilly et al., 1988); (Baudrit, 1997) mais également dans des situations de collaboration à distance, médiatisées par les TICE (Stacey, 1998); (Yaverbaum et Ocker, 1998). Dans son investigation de la réalité de l'apprentissage coopérant, Pléty (Pléty, 1996) observe des effets positifs pour la majorité des élèves en classe de mathématiques et conclut que le travail de groupe a bien des conséquences bénéfiques sur l'apprentissage. L'interaction de corésolution entre enfants serait source de progrès cognitif pour les différents partenaires, la dynamique interactive conduisant à la confrontation des points de vue et à des perturbations dans les modes de résolution individuelle, créant ainsi des déséquilibres cognitifs intra et interindividuels (Doise, 1993). Un 
tel point de vue rejoint celui développé par Vygotski (Vygotski, 1934) qui introduit la dimension sociale dans le développement intellectuel en donnant un rôle causal aux coordinations interindividuelles dans le développement cognitif.

Pour vérifier l'efficacité du conflit socio-cognitif, les auteurs ont comparé les effets de l'apprentissage collectif par rapport à l'apprentissage individuel. Pour cela, les élèves travaillent en dyades (ou en groupe) ou seuls (condition contrôle). Le développement cognitif est évalué par la différence entre un prétest et un post-test où chaque enfant travaille seul.

Toutefois, pour certains auteurs, le conflit socio-cognitif ne parait pas indispensable pour que des progrès individuels se réalisent. Des résultats allant dans ce sens sont obtenus dans des protocoles de coélaboration, de coopération, ou encore de tutelle, de co-construction et d'imitation. Selon Gilly (Gilly, 1993), "pour être efficaces, les interventions de l'autre doivent perturber les modes individuels de résolution au moment même de leur mise en œuvre. [...] Il n'est pas obligé que les interactions s'organisent sur un mode socio-conflictuel pour qu'elles provoquent des déstabilisations bénéfiques" (p. 674). Un tel changement de point de vue a conduit à focaliser les études sur les conditions et les mécanismes intervenant en cours des interactions rendant compte de la construction de compétences cognitives relatives à des classes particulières de problèmes. Ainsi, Pontecorvo (Pontecorvo, 1988) a analysé les interactions verbales d'élèves travaillant sur un mode collaboratif, et ce, dans un contexte écologique (au sein de la classe). Elle a souligné l'importance de la dimension argumentative dans la construction des connaissances dans des contextes de travail en groupe. Ce type de dispositif présente l'intérêt d'une bonne validité écologique, mais également celui de s'inscrire dans une perspective fonctionnelle, par l'analyse séquentielle des interactions : les activités cognitives et sociales des sujets sont découpées en séquences fonctionnelles.

Un des déterminants de ces interactions entre pairs est le lien affectivo-relationnel qui semble créer un contexte favorable à la collaboration et à l'acquisition de connaissances. Les amis, du fait de leur familiarité, de leurs intérêts communs et des connaissances qu'ils partagent, sont susceptibles d'établir plus aisément que les non-amis un terrain commun favorable à l'intersubjectivité, ce qui facilite le dialogue nécessaire à la coopération et aux changements cognitifs. Par exemple, Carbones i Fleta et Cuisinier (Carbones i Fleta et Cuisinier, 2003) ont étudié le rôle des relations amicales dans les interactions de résolution de problèmes en coopération auprès d'enfants âgés de 9 ans, afin d'étudier en quoi la tonalité affective de la relation modifie l'interaction elle-même ; elles ont mis au point un système d'analyse des interactions prenant en compte les modes d'échange et de gestion de la collaboration. Son utilisation pour rendre compte des interactions au sein de deux groupes de quatre enfants, l'un à tendance amicale, l'autre à tendance non amicale montre que les enfants organisent différemment leurs interactions selon qu'ils travaillent avec des amis ou des non-amis. Les enfants amis privilégient aussi bien la gestion de la relation que la gestion de la tâche. Le groupe à tendance non amicale se centre beaucoup plus sur la tâche que sur la gestion de la relation. La collaboration repose sur des processus de coopération divers chez les amis ; en revanche, elle repose presque exclusivement sur des mécanismes de confrontation chez les non amis, ce qui se traduit par un niveau de réalisation moins élevé.

D'autres déterminants des interactions lors des activités en collaboration ont été investigués comme la mixité, le niveau de compétence relative à la tâche proposée et la sociabilité. Par exemple, Da Silva (Da

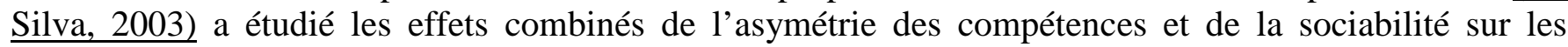
échanges lors de la co-résolution du problème. Ses résultats montrent que les enfants sociables s'assurent d'un accord mutuel régulièrement lors de la résolution alors que les enfants peu sociables utilisent fréquemment les injonctions. D'autre part, dans les dyades d'enfants sociables, l'enfant compétent sollicite et informe l'autre enfant, et leurs échanges visent une réduction de l'asymétrie initiale, alors que chez les enfants peu sociables, Da Silva (ibid.) a observé des tentatives de domination de la part de l'enfant compétent, et leurs échanges tendent au contraire à renforcer l'asymétrie initiale.

Les travaux cités montrent l'importance de prendre en compte les caractéristiques des élèves du point de 
vue des relations qu'ils entretiennent, puisque celles-ci affectent la qualité des interactions. C'est pourquoi dans les études présentées ici, nous avons utilisé un questionnaire sociométrique afin de pouvoir composer des dyades équivalentes au plan des relations, entre les différentes conditions de communication comparées.

\subsection{Apprentissage, interactions et TICE}

De nombreuses recherches ont été menées dans le but d'ouvrir une réflexion sur ce que les TICE apportent à l'apprentissage, vu sous l'angle des interactions humaines.

Avec les systèmes disponibles actuellement, la communication s'effectue le plus souvent via un support écrit, ce qui modifie les contextes de communication considérés jusqu'alors en situation de travail collectif. Alors qu'à l'oral, les interactions dialogiques aident le locuteur à activer ses connaissances et à gérer son discours (Scardamalia et Bereiter, 1986), à l'écrit la tâche est plus difficile, faute d'un interlocuteur présent. Le travail de groupe utilisant l'ordinateur crée alors les feedback nécessaires à la compréhension mutuelle par l'interaction écrite. Cependant, l'alternance entre les moments de rédaction du travail et les interventions à propos du travail concourt à un ajustement progressif des buts et des contenus. L'utilisation de l'ordinateur, dans ce cas, permet d'organiser les interactions et de favoriser le travail collaboratif (Crinon et al., 2002). Lehtinen et al. (Lehtinen et al., 1998) soulignent l'efficacité des activités collaboratives avec l'ordinateur : l'ordinateur favorise les interactions, les outils de communication rendant plus denses les échanges et permettant des feedback immédiats. Les exemples de situations où l'ordinateur sert de support à des projets de communication sont nombreux ; Crinon (Crinon, 1993) évoque un travail de création de récits par des élèves du cycle 3 destinés à des enfants d'école maternelle. Les plus jeunes "commandaient" des histoires par messagerie électronique, et les élèves de classe élémentaire écrivaient les récits et les mettaient en ligne sur le serveur télématique. Les élèves de maternelle, aidés d'un adulte, les lisaient et réagissaient; les élèves de primaire modifiaient éventuellement les histoires au regard des réactions reçues. Ce dispositif permettait donc de provoquer des discussions "collaboratives" autour des textes. Les échanges ont été nombreux et ont suscité une curiosité accrue chez les élèves de maternelle concernant l'écrit. Ce protocole a également engendré des discussions animées chez les plus grands, soit pour trouver les moyens d'intégrer les remarques faites, soit au contraire pour défendre leur point de vue. L'intérêt de ce type de dispositif est également d'ordre social : l'auteur a constaté une forte motivation chez les élèves, une grande satisfaction à l'égard du travail réalisé, et surtout un grand intérêt pour le sujet, associé à un accroissement des compétences d'écriture. Cette étude utilisait la télématique, mais aujourd'hui l'utilisation d'Internet permet de communiquer en temps réel. Barbier et al. (Barbier et al., 1993) ont ainsi souligné les effets positifs du travail en réseau sur la production de texte. Le réseau favoriserait la communication et les interactions entre pairs et améliorerait leurs compétences à planifier et à réviser des textes ; en d'autres termes, les interactions sont favorables à l'atteinte du but rédactionnel. En effet, l'écriture "papier-crayon" est le plus souvent individuelle et privée, alors que le caractère "public" de l'écrit sur écran favorise les interactions entre élèves (Hawisher et Selfe, 1999). Zammuner (Zammuner, 1995) a ainsi comparé les récits d'élèves réalisés sur traitement de texte dans trois conditions : 1) écriture et révision individuelles ; 2) écriture individuelle et révision avec un pair et 3) écriture et révision avec un pair. L'auteur a constaté des améliorations lors de la révision dans les conditions 1 et 3 , mais ces améliorations ne concernent qu'un nombre restreint de critères. En revanche, dans la condition 2, les révisions opérées sont plus importantes et concernent quasiment tous les critères. Le brouillon collaboratif est également meilleur que le brouillon individuel, ce qui assoit une fois de plus la supériorité du travail collaboratif par rapport au travail individuel lors d'activités pédagogiques. Ce type d'étude montre également l'intérêt d'alterner le travail individuel et le travail collaboratif, idée que nous avons retenue pour nos études.

Si la plupart des auteurs s'accordent sur le fait que le travail collaboratif est plus efficace que le travail individuel, peu d'études à notre connaissance se sont centrées sur la collaboration écrite, synchrone et à distance. En effet, dans un tel contexte, plusieurs questions se dégagent : 1) en quoi l'usage de l'écrit dans 
des contextes d'interaction synchrone à distance modifie le contenu des échanges et la structure des échanges ? ; 2) en quoi des différences relatives à la maîtrise de l'écrit affectent-elles les échanges entre les interactants?

Nous supposons que le fait de collaborer par écrit et à distance engendrera moins d'échanges que lors d'une situation de communication en face à face, mais que leur contenu sera plus ciblé sur la tâche à accomplir. Par ailleurs, nous supposons que le niveau de maîtrise de l'écrit (dans notre étude, nous comparons des étudiants d'université à des collégiens) aura une incidence sur le nombre et la qualité des échanges.

\section{Le contexte de collaboration}

Dans nos études, les participants sont mis dans une situation de collaboration à distance via un outil informatique, en dyades. Les dyades ont une tâche commune : construire une carte conceptuelle à partir d'un texte. Chacun a auparavant réalisé cette tâche individuellement, ce qui assure d'une part que tous les participants à l'étude ont déjà procédé à une lecture attentive du texte, et d'autre part, comme nous l'avons vu précédemment, cela place les élèves dans des conditions optimales de travail. Les élèves, disposant des cartes conceptuelles produites individuellement, doivent s'accorder sur la meilleure carte conceptuelle possible et la construire à l'aide d'un logiciel : ModellingSpace.

\subsection{L'environnement informatique : Le logiciel ModellingSpace}

L'environnement informatique utilisé est le logiciel ModellingSpace dans sa version 1.02 élaboré et conçu dans le cadre du projet européen homonyme de l'IST-School of Tomorrow (IST-2000-25-585) ${ }^{\underline{1}}$. Ce logiciel est issu du développement de ModelsCreator (Komis et al., 2001) conçu initialement pour des élèves âgés de 11 à 16 ans; il permet de créer des modèles sur la base d'un environnement ouvert permettant de travailler sur des entités et sur leurs propriétés qui se lient par des relations d'ordre qualitatif, semi-quantitatif ou quantitatif. Cet environnement éducatif permet la construction individuelle et/ou en commun de différentes formes de modèles. Un modèle est un système d'entités qui sont reliées, soit par des relations sémantiques ou logiques (cas des cartes conceptuelles), soit semi-quantitatives (relation de co-variation par exemple), ou quantitatives (relations mathématiques). Il inclut des outils qui permettent de créer et d'éditer des entités et de construire et d'explorer des modèles à partir de ces entités. Il permet le travail individuel ou en collaboration en rendant possible les interactions synchrones comme asynchrones entre les élèves qui travaillent ensemble. Ainsi, les élèves peuvent être de la même classe ou d'écoles ou même de pays différents. Enfin, il est pourvu d'outils d'analyse de la construction des modèles et des interventions entre élèves (Dimitracopoulou, 2003).

Le logiciel est conçu pour que les partenaires distants collaborent principalement en partageant le modèle sur l'espace de travail qui devient ainsi un espace cognitif (Margaritis et al., 2003). La synchronie est assurée, et l'action d'un participant sur un des objets partagés est visible par son ou ses partenaire(s) en temps réel. Pour une meilleure coordination de l'activité des partenaires, le logiciel est équipé d'un système de clé rendant possible à son possesseur et seulement à lui l'action sur les objets partagés pendant que ses partenaires peuvent observer ses actions. La gestion de la possession de la clé est assurée par un système de "demande de clé", "passage de clé", de "clé acceptée" et de "clé refusée" ou de "demande refusée". Par ailleurs, un outil de dialogue en direct ou chat est intégré au logiciel. Il est en outre doté d'"ouvreurs de dialogues", c'est-à-dire de phrases types, préenregistrées pouvant être ou non activées. Ainsi, des messages écrits peuvent être échangés par les partenaires tout au long de la collaboration.

La figure 1 présente l'espace de travail proposé aux utilisateurs du logiciel dans le contexte des études présentées dans cette recherche. 


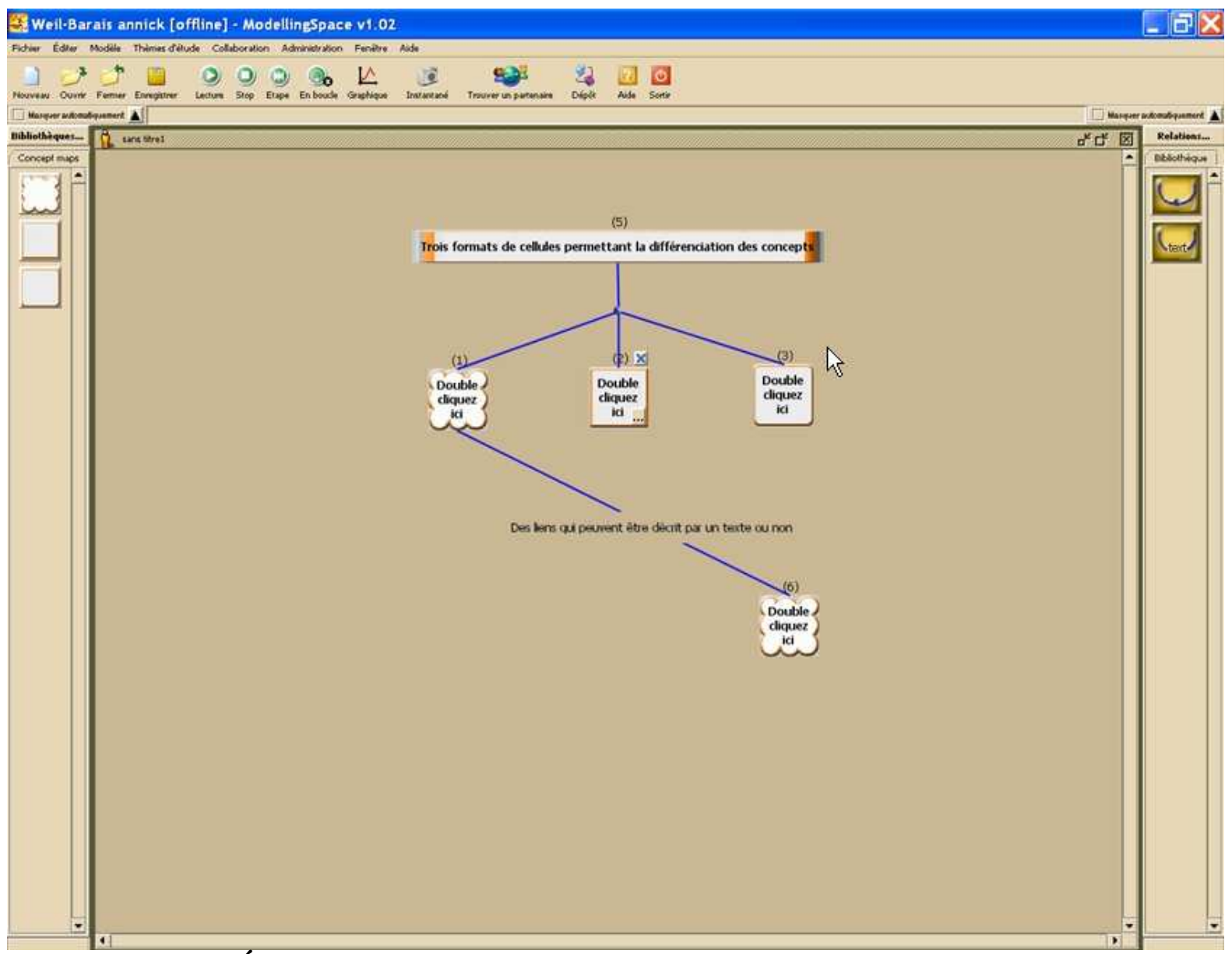

Figure 1 - Écran représentant l'espace de travail du logiciel ModellingSpace

De part et d'autre de la "table de travail" où les éléments sont insérés pour former peu à peu la carte conceptuelle, les utilisateurs trouvent les bibliothèques d'entités (à leur gauche) et de relations qualitatives (à leur droite). Ils ont à leur disposition trois formats de cellule pour les entités permettant de différencier les concepts, et deux types de relation : lien simple et lien décrit par un texte. Pour insérer une entité ou un lien, il suffit de cliquer sur l'objet, et de le faire glisser jusqu'à l'emplacement décidé sur l'espace de travail. Pour insérer du texte dans une cellule d'entité ou un lien, l'utilisateur doit doublecliquer où il lui est indiqué de le faire, et le curseur apparaît en mode édition. Pour valider son écrit, il suffit de cliquer à l'extérieur de l'entité. Chaque objet peut être déplacé, modifié ou supprimé d'un simple clic. Un lien est établi entre deux ou plusieurs entités lorsque l'utilisateur les a sélectionné en cliquant sur elles puis n'importe où sur l'espace de travail lorsqu'il a terminé.

Lorsque l'utilisateur lance une recherche de partenaire (en cliquant sur l'icône de la barre d'outils), la liste des membres de son groupe apparaît et il lui est spécifié lesquels d'entre eux sont connectés au même réseau que son ordinateur (et par conséquent à même de travailler en collaboration avec lui). Il doit sélectionner le membre du groupe auquel il souhaite envoyer une proposition de collaboration. Son partenaire reçoit un avis, et accepte (ou non) l'invitation.

L'utilisation de la clé permet de limiter les interventions sur l'espace de travail à son seul détenteur. Une colonne "collaboration" à gauche indique lequel des partenaires identifiés possède la clé. Seul l'utilisateur qui a la clé peut agir sur l'espace de travail. Le partenaire visualise le même écran que lui, et suit ainsi les modifications instantanément. Il a la possibilité de demander la clé ; libre au destinataire de la demande de la lui passer ou de la conserver. Les utilisateurs ont l'opportunité de communiquer à tout moment via le chat, situé en dessous de l'écran. 
Ce logiciel présente l'intérêt d'être pourvu d'un outil d'analyse (Supervision Tool v1.02) des modèles produits. En effet, toutes les actions du ou des constructeurs du modèle sont sauvegardées dans un fichier annexe appelé "fichier historique". Tout l'historique de la construction du modèle y est consultable. Les différents écrans associés à cet outil sont présentés et commentés ci-après.

La figure 2 montre l'écran qui fournit une vue d'ensemble des différents éléments de l'activité de résolution en différents tableaux et cadres: la détention de la clé, des informations qualitatives et quantitatives sur les entités et les relations du modèle, un tableau récapitulatif des actions des partenaires, et le détail du dialogue du chat à droite de l'écran.

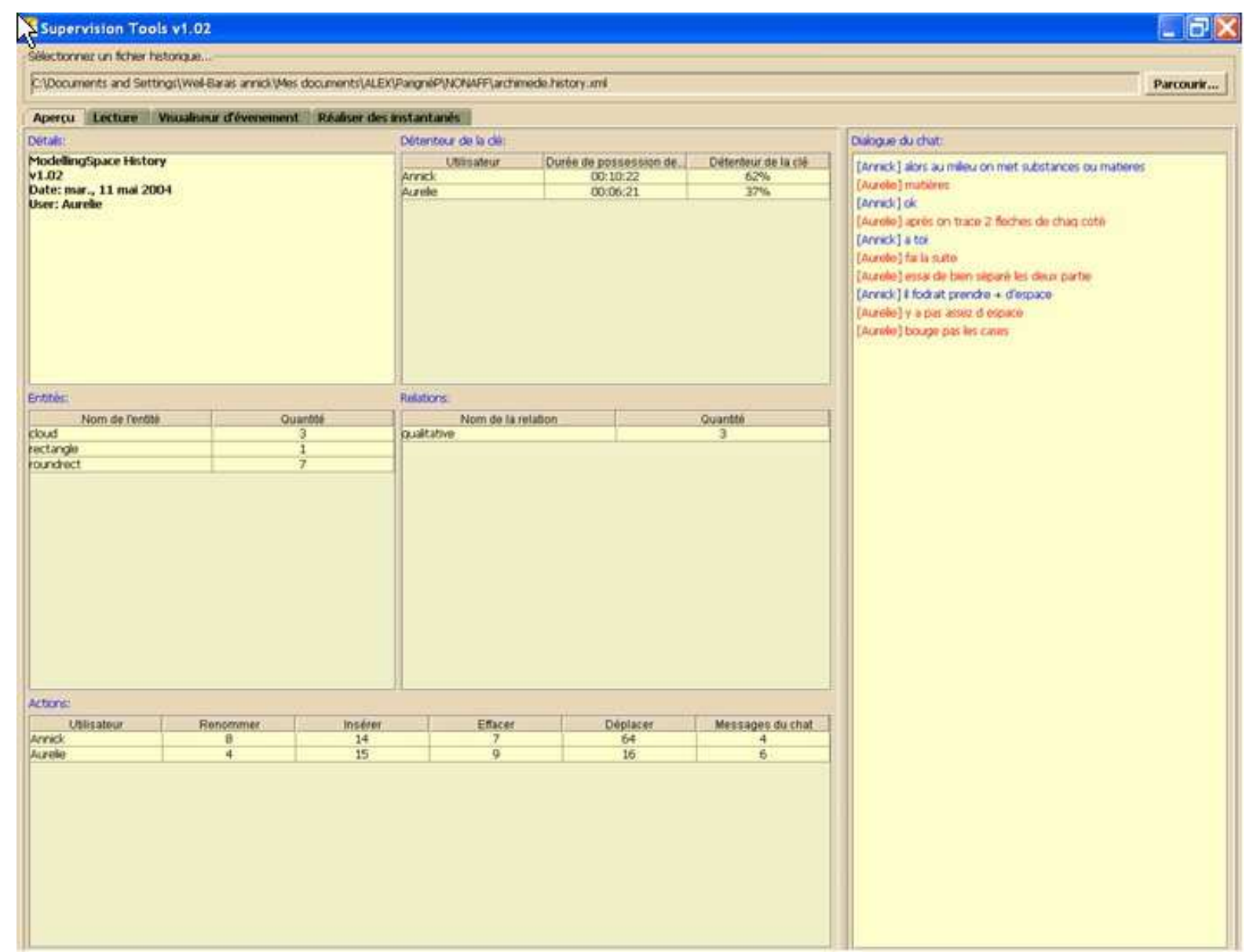

Figure 2 - Ecran d'aperçu du fichier historique

La figure 3 illustre la modalité d'accès à la fonction de lecture de la construction du modèle. Dans les trois colonnes de la partie supérieure de l'écran, la construction du modèle défile : l'espace partagé occupe la plus grande partie de l'écran, puis les objets supprimés arrivent à mesure dans la colonne du milieu et à droite, les dialogues du chat apparaissent à mesure. Une description de chaque action est donnée dans le cadre du bas. 


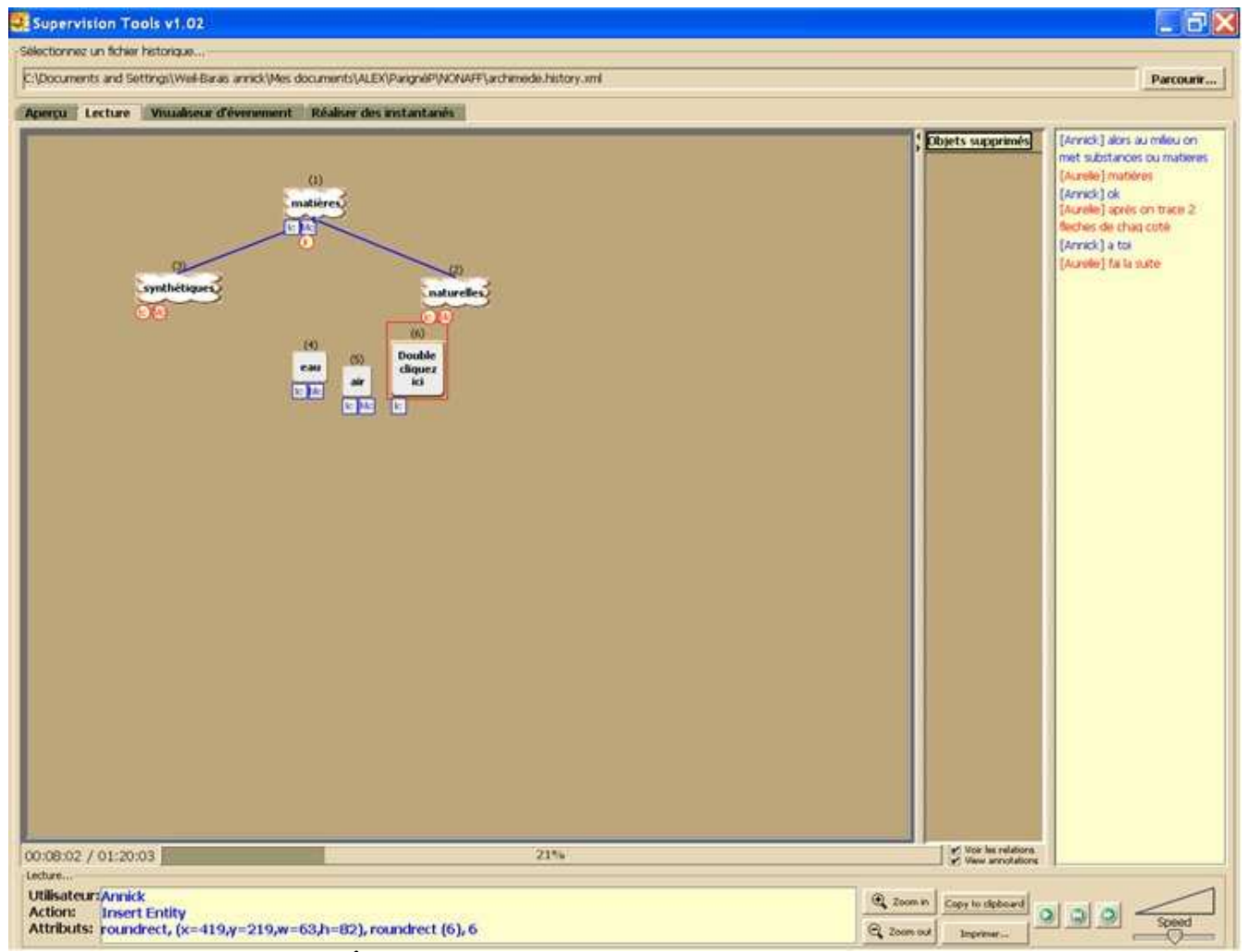

Figure 3 - Écran de lecture de la progression du modèle

Enfin, le tableau de la figure 4 fournit le détail de toutes les actions, inscrites dans le temps absolu et relatif. Il permet de repérer l'activité propre à chaque utilisateur. Chaque action est répertoriée (message du chat, insertion d'une entité, passage de la clé...).

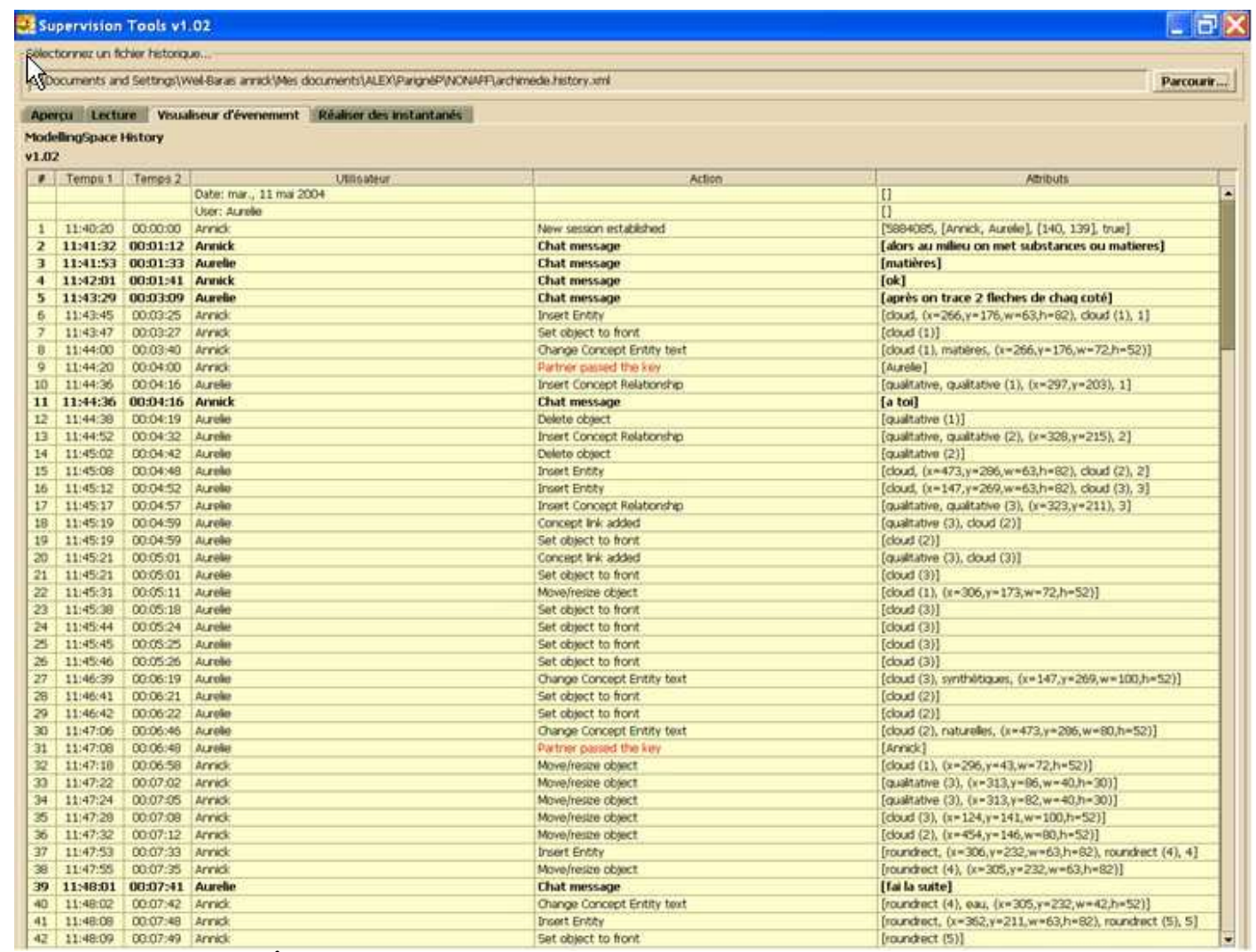

Figure 4 - Écran de visualisation des actions produites sur le modèle 


\subsection{La tâche : construction d'une carte conceptuelle à partir d'un texte}

Une carte conceptuelle (concept map) est une représentation graphique (donc spatialisée) d'une base de connaissances déclaratives qui possède une organisation dans laquelle les concepts sont liés entre eux par des liens (Novak et Gowin, 1984). Une carte conceptuelle est constituée de nœuds ou de cellules qui contiennent les "étiquettes linguistiques" des concepts. Les liens entre les concepts peuvent être si nécessaire décrits par un texte court. Ils se terminent parfois par des flèches qui donnent le sens de la relation entre les concepts. Novak et Gowin (ibid.) considèrent les cartes conceptuelles non comme un outil d'apprentissage mais comme un moyen d'acquérir des procédures d'apprentissage ; c'est pourquoi ils les considèrent comme un outil métacognitif. Des usages différents sont envisagés selon que les cartes sont élaborées par l'enseignant ou par les apprenants. Pour l'enseignant, elles permettent de visualiser l'articulation entre les concepts à enseigner. Pour les apprenants, elles peuvent être un moyen d'expliciter ce qu'ils connaissent sur un sujet avant de l'aborder. Elles sont également un outil de synthèse après l'apprentissage. Enfin, elles peuvent constituer le moyen de représenter et de s'approprier le contenu d'un texte.

Certains systèmes hypermédias possèdent des outils graphiques, dans le but de permettre à l'apprenant de construire des représentations spatiales structurées. Ces outils, qui permettent à l'apprenant de dessiner des graphiques, des diagrammes ou des liens entre les concepts (et ainsi créer des cartes conceptuelles), sont supposés conduire les apprenants à expliciter leur propre activité d'apprentissage en facilitant la création de relations sémantiques entre les informations d'une part et en favorisant la construction d'un modèle mental du domaine étudié d'autre part (Goldman, 1996). Selon Jonassen et al. (Jonassen et al., 1998), la carte conceptuelle est un outil cognitif, puisqu'elle est à la fois le résultat et l'outil de la construction des relations entre les concepts. Ces relations expriment les connaissances sur la structuration du domaine. Les outils d'aide à la création de cartes conceptuelles seraient ainsi des environnements d'apprentissage à part entière (Mc Aleese, 1998).

La tâche de production d'une carte conceptuelle a été choisie parce qu'elle nous permettait de réaliser notre étude dans différents contextes éducatifs pertinents à la fois pour des élèves de collège et des étudiants d'université. Rappelons que la lecture analytique de texte est une des compétences visées par les programmes d'enseignement de $4^{\text {ème }}$. Le texte proposé aux collégiens a été élaboré par l'enseignant et constitue un support de son enseignement. Pour les étudiants d'université (niveau licence), le texte a été choisi en fonction du thème abordé par l'enseignant.

Il est possible de produire plusieurs cartes conceptuelles à partir d'un texte; il n'y a donc pas de réponse canonique attendue, ce qui est propice aux interactions. L'utilisation du logiciel ModellingSpace pour la construction des cartes conceptuelles, compte tenu des outils disponibles décrits précédemment, nous a grandement facilité le recueil et le traitement des données.

\section{Présentation des études}

Deux études ont été conduites. La première étude vise à déterminer en quoi l'usage de l'écrit dans des contextes d'interaction synchrone à distance modifie le contenu et la structure des échanges chez des étudiants universitaires. Nous comparons les échanges au sein de petits groupes composés de deux étudiants (désignés dans la suite de cet article par "dyades"), dans deux situations de communication - orale en face à face, écrite à distance via un dispositif informatique - lors d'une séance de travail collaboratif. Dans la deuxième étude, nous avons répliqué la modalité de communication à distance chez des collégiens âgés de 14 ans en moyenne, et nous avons comparé les contenus et les structures des échanges à ceux de notre population universitaire. 


\subsection{Méthode}

\subsubsection{Population}

La première étude a été menée auprès de 22 étudiants de troisième année de psychologie de l'Université d'Angers, tous issus d'un même groupe de travaux dirigés (T.D.). La seconde étude a été menée auprès de 16 élèves d'une classe de $4^{\text {ème }}$, âgés en moyenne de 14 ans (écart-type $=7$ mois 2 jours ; min. $=13$ ans 6 mois ; max. $=15$ ans 4 mois). Tous les participants étaient volontaires et consentants.

Sachant l'importance que peuvent avoir les relations amicales sur la communication entre pairs (Carbones i Fleta et Cuisinier, 2003), nous avons choisi de contrôler la variable « affinité » en répartissant les dyades affinitaires et non affinitaires dans les deux conditions de communication. Pour ce faire, nous avons réalisé au sein de chaque groupe impliqué dans l'étude (groupe de T.D. à l'université et classe de $4{ }^{\text {ème }}$ en collège) une enquête sociométrique : chaque étudiant et chaque élève devait choisir parmi ses pairs ceux avec lesquels il souhaitait travailler et ceux avec lesquels il ne souhaitait pas travailler parmi la liste des membres de la classe (ou du groupe de TD). Ainsi, nous avons constitué des dyades affinitaires (i.e. dont les deux interlocuteurs se sont choisis mutuellement) et non affinitaires (i.e. dans lesquelles les deux partenaires ne se sont ni choisis, ni exclus). Le détail de la constitution des dyades figure au tableau 1 cidessous.

\begin{tabular}{|l|l|l|l|}
\hline & $\begin{array}{l}\text { Etude 1 } \\
\text { (Étudiants universitaires) }\end{array}$ & $\begin{array}{l}\text { Etude 2 } \\
\text { (Collégiens) }\end{array}$ \\
\hline \hline $\begin{array}{l}\text { Communication } \\
\text { Type de dyade }\end{array}$ & $\begin{array}{l}\text { Communication } \\
\text { face à face }\end{array}$ & $\begin{array}{l}\text { Communication } \\
\text { à distance }\end{array}$ & $\begin{array}{l}\text { Communication } \\
\text { à distance }\end{array}$ \\
\hline \hline Affinitaires & 3 & 3 & 4 \\
\hline \hline Non affinitaires & 2 & 3 & 4 \\
\hline \hline Total & $\mathbf{5}$ & $\mathbf{6}$ & $\mathbf{8}$ \\
\hline \hline
\end{tabular}

Tableau 1 - Répartition du nombre de dyades selon leur caractère affinitaire ou non affinitaire pour chaque étude

\subsubsection{Matériel}

Le texte à partir duquel les étudiants universitaires ont réalisé leur carte conceptuelle est un article intitulé "Interaction", extrait d'un ouvrage à caractère encyclopédique ${ }^{2}$. En ce qui concerne les élèves de la deuxième étude, il s'agissait d'un texte explicatif et descriptif de la synthèse des produits et de son évolution aux travers des âges ${ }^{\underline{3}}$. Avant d'accomplir la tâche demandée, les participants ont été informés de ce qu'est une carte conceptuelle. Une définition adaptée aux différents publics a été donnée ainsi que des exemples (un pour les étudiants universitaires et deux pour les collégiens). Pour les collégiens, le premier exemple présenté est la carte conceptuelle du mot "poisson" construite à partir de la définition du mot donnée dans le Petit Larousse Illustré (1996). Sous la carte, la définition est recopiée et les concepts intégrés dans la carte y sont soulignés. Les concepts sont différenciés par l'utilisation de différents encadrés. Le deuxième exemple est la carte proposée à titre d'exemple par (Tribollet et al. 1997) à propos du phénomène de l'ébullition. Il s'agit d'une carte plus complexe, où les liens sont décrits par un texte. L'exemple proposé aux étudiants concernait le concept même de carte conceptuelle. 


\subsubsection{Procédure}

Pour les deux études, la recherche s'est déroulée en trois phases : une phase au cours de laquelle les participants sont initiés collectivement aux cartes conceptuelles, une phase où ils doivent en réaliser une individuellement à partir d'un texte au moyen d'outils classiques (papier, gomme et crayon) et enfin une phase de travail collaboratif où ils doivent réaliser la carte qui leur semble la meilleure possible, en utilisant le logiciel ModellingSpace.

Dans les deux études, dans la phase de collaboration, les participants avaient à leur disposition le texte de référence, leur propre production et celles de leur binôme. Dans tous les cas, la dernière phase de l'expérimentation est lancée lorsque les participants maîtrisent l'utilisation du logiciel, lequel leur est présenté en début de séance de travail (création de concepts, de liens, utilisation de la clé...).

\subsection{Analyse des résultats}

Bien que notre intérêt se porte principalement sur les interactions au sein des dyades lors de la réalisation de la carte conceptuelle, nous avons d'abord réalisé, pour la première étude, une analyse de la structure formelle des cartes conceptuelles de chacune des dyades composées d'étudiants ${ }^{4}$.

Il s'avère que, dans les deux conditions de collaboration, la structure des cartes produites est comparable. En effet, dans l'étude 1, nous comptabilisons en moyenne 5,6 concepts centraux sur les cartes conceptuelles en condition de communication en face à face et 5,2 concepts centraux en condition de communication à distance. De la même manière, le nombre de liens sur les cartes varie peu (20,6 liens en moyenne sur les cartes en communication en face à face versus 21,8 liens en communication à distance). Les cartes réalisées en face à face comptent en moyenne 5,6 composantes principales du concept faisant l'objet d'une présentation dans l'article (l'interaction), les cartes en condition de travail à distance en comptent 5,2.

\subsubsection{Analyse des interactions selon le mode de communication}

Si la comparaison des cartes conceptuelles produites en collaboration ne montre pas de différence selon la modalité de communication, nous nous interrogeons sur la qualité de la communication. L'analyse a été conduite à partir de la retranscription des verbalisations enregistrées au magnétophone pour les dyades communiquant en face à face et à partir des logfiles enregistrés dans les fichiers historiques du logiciel ModellingSpace pour les dyades communiquant via le chat (figure 2, pour une visualisation des fichiers historiques du logiciel). Nous avons par la suite distingué dans les analyses le nombre d'interventions du nombre d'échanges. Un échange est caractérisé par un ensemble d'interventions sous-tendues par un même but. Nous opérerons d'abord une analyse quantitative des interactions, puis une analyse qualitative, portant sur le contenu même des échanges.

\subsubsection{Analyse quantitative}

Parmi les multiples critères d'analyse dont dispose le logiciel ModellingSpace grâce à la consultation du fichier historique de chaque dyade, quatre indicateurs ont été retenus. Il s'agit d'abord de la durée totale de l'interaction, ensuite du nombre d'interventions et du nombre d'échanges et enfin du nombre d'actions opérées sur le modèle durant la session de travail. Pour chacun des contextes de communication, les moyennes et écarts-types de ces indicateurs sont indiqués dans le tableau 2. 


\begin{tabular}{|c|c|c|c|}
\hline & & $\begin{array}{l}\text { Communication } \\
\text { face à face }\end{array}$ & $\begin{array}{l}\text { Communication } \\
\text { à distance }\end{array}$ \\
\hline \multirow{2}{*}{$\begin{array}{ll}\text { durée } & \text { de } \\
\text { l'interaction } & \\
\text { (en minutes) } & \end{array}$} & moyenne & 66,8 & 80,8 \\
\hline & $\begin{array}{l}\text { écart } \\
\text { type }\end{array}$ & 15,6 & 9,9 \\
\hline \multirow{2}{*}{$\begin{array}{l}\text { nombre total } \\
\text { d'interventions }\end{array}$} & moyenne & 480,6 & 81,8 \\
\hline & $\begin{array}{l}\text { écart } \\
\text { type }\end{array}$ & 75 & 42 \\
\hline \multirow{2}{*}{ nombre d'échanges } & moyenne & 120,6 & 37,7 \\
\hline & $\begin{array}{l}\text { écart } \\
\text { type }\end{array}$ & 14,3 & 14,3 \\
\hline \multirow{2}{*}{$\begin{array}{l}\text { nombre d'actions } \\
\text { opérées } \\
\text { sur le modèle }\end{array}$} & moyenne & 467,8 & 691,2 \\
\hline & $\begin{array}{l}\text { écart } \\
\text { type }\end{array}$ & 108 & 130 \\
\hline
\end{tabular}

Tableau 2 - Moyenne et écart-type de chaque critère d'analyse selon le mode de communication (étude 1)

La durée totale de l'interaction varie en fonction du mode de communication : il faut en effet quasiment un quart d'heure de plus aux dyades communiquant via le chat pour réaliser leur modèle $(80,8$ minutes en moyenne) par rapport à ceux communiquant en face à face (66,8 minutes en moyenne, soit un écart de 14 minutes), cet écart étant probablement dû au fait que les dyades communiquant via le chat doivent rédiger leurs échanges au clavier.

Le nombre total d'interventions est nettement supérieur pour les dyades communiquant en face à face (480,6 interventions en moyenne), alors que l'on recense en moyenne 81,8 interventions pour les dyades communiquant à distance. De la même manière, le nombre d'échanges de ceux qui communiquent en face à face est nettement supérieur à ceux qui communiquent via le chat (respectivement 120,6 et 37,7 en moyenne).

Concernant le nombre d'actions opérées sur le modèle, nous constatons que c'est cette fois les dyades communiquant à distance qui manipulent le plus l'espace de travail avec un nombre moyen d'actions s'élevant à 691,2, alors que 467,8 actions en moyenne sont enregistrées chez les dyades communiquant en face à face.

Pour résumer, cette première analyse nous indique que les dyades communiquant à distance mettent plus de temps à réaliser leur carte conceptuelle, mais échangent moins et manipulent plus que les dyades communiquant en face à face. La nature des échanges peut toutefois nous donner des indicateurs supplémentaires sur le mode de collaboration en fonction de leurs situations de communication.

En adoptant la typologie des échanges de (Kerbrat-Orecchioni, 1990), nous distinguerons dans ce second point de l'analyse quantitative les échanges tronqués, restreints et étendus. Les échanges tronqués ne comptent qu'une seule intervention d'un des deux interlocuteurs, sans que cette intervention n'entraîne de réaction de la part de l'autre interlocuteur. Les échanges restreints comptent deux interventions seulement, et les échanges étendus comptent au moins trois interventions, sous tendues par le même but, 
manifestant une co-construction en collaboration des deux interlocuteurs. Sans entrer dans le détail des raisons théoriques qui nous poussent à utiliser cette typologie, l'étendue des échanges nous semble constituer un indicateur pertinent de l'attention conjointe entre les membres d'un binôme. C'est à ce titre que nous l'avons préférée à d'autres modèles comme celui des actes de langage, mieux adapté pour rendre compte des rôles et des places des interactants (voir l'usage qui en est fait par George, 2003). Le tableau 3 indique les moyennes et répartitions des types d'échanges dans les deux conditions de communication.

\begin{tabular}{|l|l|l|l|l|}
\hline & \multicolumn{2}{|l|}{$\begin{array}{l}\text { Communication } \\
\text { face à face }\end{array}$} & \multicolumn{2}{l|}{$\begin{array}{l}\text { Communication } \\
\text { à distance }\end{array}$} \\
\hline \hline & $\begin{array}{l}\text { Moyenne } \\
\text { (écart- } \\
\text { type })\end{array}$ & $\begin{array}{l}\text { Proportion } \\
(\text { en \%) }\end{array}$ & $\begin{array}{l}\text { Moyenne } \\
\text { (écart- } \\
\text { type })\end{array}$ & $\begin{array}{l}\text { Proportion } \\
(\text { en \% })\end{array}$ \\
\hline \hline $\begin{array}{l}\text { Echanges } \\
\text { tronqués }\end{array}$ & $\begin{array}{l}22,2 \\
(8,1)\end{array}$ & 18,4 & $\begin{array}{l}176,67 \\
(8,3)\end{array}$ & 44,2 \\
\hline \hline $\begin{array}{l}\text { Echanges } \\
\text { restreints }\end{array}$ & $\begin{array}{l}25,4 \\
(7)\end{array}$ & 21,1 & $\begin{array}{l}11,5 \\
(1,4)\end{array}$ & 30,5 \\
\hline \hline \hline $\begin{array}{l}\text { Echanges } \\
\text { étendus }\end{array}$ & $\begin{array}{l}73 \\
(13,7)\end{array}$ & 60,5 & $\begin{array}{l}9,50 \\
(6,8)\end{array}$ & 25,2 \\
\hline \hline
\end{tabular}

Tableau 3 - Nombre moyen, écart-type et proportion d'échanges tronqués, restreints et étendus pour chaque groupe expérimental (étude 1)

Nous observons tout d'abord que les échanges tronqués ${ }^{\underline{5}}$ sont plus nombreux pour les dyades communiquant via le chat (44,2\% des échanges) que pour ceux communiquant verbalement $(18,4 \%$ des échanges) : $\mathrm{X}^{2}(\mathrm{ddl} 1, \mathrm{~N}=11)=15,52 ; \mathrm{p}<.01$. Les proportions d'échanges restreints sont équivalents dans les deux conditions de coopération $(21,1 \%$ pour les dyades communiquant en face à face et 30,5\% pour ceux à distance : $\mathrm{X}^{2}=2,34$ ). Enfin, la proportion d'échanges étendus (plus de trois interventions) est cette fois nettement supérieure pour les dyades communiquant en face à face $(60,5 \%$ des échanges) en comparaison avec ceux communiquant à distance $\left(25,2 \%\right.$ des échanges) : $\mathrm{X}^{2}(\mathrm{dd} 11, \mathrm{~N}=11)=25,45$; $\mathrm{p}<.01$. Ainsi, le mode de communication semble directement influer sur le type d'échanges : les étudiants communiquant en face à face privilégient les échanges étendus, alors que ceux communiquant à distance privilégient les échanges tronqués.

\subsubsection{Analyse qualitative : objet des échanges}

En nous inspirant de la classification des actes de parole proposée par (Chabrol et Bromberg, 1999), nous avons développé une grille d'analyse permettant de classifier les échanges enregistrés dans notre étude selon leur contenu. Globalement, les échanges se répartissent dans une dizaine de catégories, dont nous avons consigné quelques exemples dans le tableau 4. 


\begin{tabular}{|c|c|c|c|}
\hline \multirow{3}{*}{ CARTE } & $\begin{array}{l}\text { mise } \\
\text { forme }\end{array}$ & 1 & $\begin{array}{l}\text { remonte la carte/ décale un peu } \\
\text { cognition vers la gauche }\end{array}$ \\
\hline & liens & 2 & $\begin{array}{l}\text { mets une flèche entre ordre social } \\
\text { et lien social/on devrait liẹ } \\
\text { cognition et apprentissage je } \\
\text { pense }\end{array}$ \\
\hline & concept & 3 & $\begin{array}{l}\text { de l'interaction émergent les } \\
\text { formes sociales, linguistiques et } \\
\text { cognitives/l'interaction est une } \\
\text { relation intersubjective }\end{array}$ \\
\hline LOGICIEL & utilisation & 4 & $\begin{array}{l}\text { comment je fais pour écrire dans } \\
\text { les carrés?/ comment on fait les } \\
\text { liens déjà? }\end{array}$ \\
\hline \multirow{2}{*}{ TACHE } & organisation & 5 & $\begin{array}{l}\text { on commence par regarder nos } \\
\text { cartes pour voir ce qu'il y a de } \\
\text { commun et je t'envoie un message } \\
\text { après/ on n'a qu'à mettre nos trois } \\
\text { domaines, et on les dévelope } \\
\text { après ok? }\end{array}$ \\
\hline & définition & 6 & $\begin{array}{l}\text { c'est un truc qu'on sait pas trop } \\
\text { faire la carte conceptuelle, t'as } \\
\text { compris quoi toi? / Non pour moi } \\
\text { c'est pas ça une carte conceptuelle } \\
\text { en fait }\end{array}$ \\
\hline TEXTE & $\begin{array}{l}\text { référence/ } \\
\text { citation }\end{array}$ & 7 & $\begin{array}{l}\text { premier } \quad \text { paragraphe } \\
\text { perspectives: soit... soit... c'est là// } \\
\text { où as tu vu ça dans le texte??? }\end{array}$ \\
\hline \multicolumn{2}{|c|}{$\begin{array}{l}\text { RELATIONS } \\
\text { SOCIALES }\end{array}$} & 8 & $\begin{array}{l}\text { tu es d'Angers toi?/ comment ça } \\
\text { va? /Tu as trouvé un stage toi au } \\
\text { fait? }\end{array}$ \\
\hline \multicolumn{2}{|l|}{ AUTRE } & 9 & $\begin{array}{l}\text { désolé, mauvaise frappe!/ Oh tu } \\
\text { dors?/ll fait carrément trop chaud } \\
\text { ici! }\end{array}$ \\
\hline \multicolumn{2}{|c|}{$\begin{array}{l}\text { ORDINATEUR (clé / } \\
\text { réalisation de la tâche) }\end{array}$} & 10 & $\begin{array}{l}\text { vas y passe la clef/ je t'ai passé la } \\
\text { clé là, vas y!/Tu veux qu'on } \\
\text { échange à l'ordinateur?/Tiens je } \\
\text { peux essayer moi aussi de faire à } \\
\text { l'ordinateur? }\end{array}$ \\
\hline
\end{tabular}

Tableau 4 - Exemples d'échanges pour chacune des catégories de la grille de classification

Les trois premières catégories se réfèrent à la carte en elle-même, et concernent 1) la mise en forme, 2) les relations entre les concepts et 3) les concepts. Une quatrième catégorie d'échanges concerne directement l'utilisation du logiciel ModellingSpace. Les deux catégories suivantes concernent la réalisation de la tâche, soit dans son organisation (planification des actions principalement), soit la notion de carte conceptuelle. Une septième classe d'échanges est liée au texte (référence, citations). La huitième catégorie d'échanges englobe toutes formes de relations sociales échangées lors de la réalisation de la tâche. Une catégorie "autre" comprend les échanges ne permettant pas d'être classés dans l'une de ces catégories (généralement, ils concernent l'expression d'affects et de sentiments). Une dixième et dernière classe d'échanges concerne la clé pour les dyades qui communiquent à distance ou bien l'utilisation de l'ordinateur pour la réalisation de la tâche dans la condition "face à face".

Chaque échange a été répertorié dans une des catégories ainsi définies. Le tableau 5 indique le nombre moyen d'échanges par catégorie, ainsi que leur répartition pour chaque condition de communication. 


\begin{tabular}{|c|c|c|c|c|c|c|}
\hline & & & \multicolumn{2}{|c|}{$\begin{array}{l}\text { Communication } \\
\text { face à face }\end{array}$} & \multicolumn{2}{|c|}{$\begin{array}{l}\text { Communication } \\
\text { à distance }\end{array}$} \\
\hline & & & $\begin{array}{l}\text { Moyenne } \\
\text { (écart- } \\
\text { type) }\end{array}$ & $\begin{array}{l}\text { Proportion } \\
\text { (en \%) }\end{array}$ & $\begin{array}{l}\text { Moyenne } \\
\text { (écart- } \\
\text { type) }\end{array}$ & $\begin{array}{l}\text { Proportion } \\
(\text { en \%) }\end{array}$ \\
\hline \multirow{3}{*}{ CARTE } & $\begin{array}{l}\text { mise } \\
\text { forme }\end{array}$ & 1 & $\begin{array}{l}21,2 \\
(6,2)\end{array}$ & 16,7 & $\begin{array}{l}3,5 \\
(2,8)\end{array}$ & 9,3 \\
\hline & liens & 2 & $\begin{array}{l}13,6 \\
(3,4)\end{array}$ & 11,6 & $\begin{array}{l}5 \\
(4,1)\end{array}$ & 13,3 \\
\hline & concepts & 3 & $\begin{array}{l}37,6 \\
(11,6)\end{array}$ & 29,6 & $\begin{array}{l}10,5 \\
(4,5) \\
\end{array}$ & 27,9 \\
\hline LOGICIEL & utilisation & 4 & $\begin{array}{l}11,2 \\
(5,5)\end{array}$ & 8,8 & $\begin{array}{l}2,7 \\
(2)\end{array}$ & 7,1 \\
\hline \multirow{2}{*}{ TACHE } & organisation & 5 & $\begin{array}{l}18,8 \\
(6,6)\end{array}$ & 14,8 & $\begin{array}{l}3,5 \\
(0,8)\end{array}$ & 9,3 \\
\hline & définition & 6 & $\begin{array}{l}1,8 \\
(2,7)\end{array}$ & 1,4 & $\begin{array}{l}0 \\
(0)\end{array}$ & $\mathbf{0}$ \\
\hline TEXTE & $\begin{array}{l}\text { référence/ } \\
\text { citation }\end{array}$ & 7 & $\begin{array}{l}10,4 \\
(3,6)\end{array}$ & 8,2 & $\begin{array}{l}1,3 \\
(2,4)\end{array}$ & 3,5 \\
\hline \multicolumn{2}{|c|}{$\begin{array}{l}\text { RELATIONS } \\
\text { SOCIALES }\end{array}$} & 8 & $\begin{array}{l}1,6 \\
(1,3)\end{array}$ & 1,3 & $\begin{array}{l}1,8 \\
(0,7)\end{array}$ & 4,9 \\
\hline \multicolumn{2}{|l|}{ AUTRE } & 9 & $\begin{array}{l}8,80 \\
(3,27)\end{array}$ & 6,94 & $\begin{array}{l}5,50 \\
(5,13)\end{array}$ & 14,60 \\
\hline \multicolumn{2}{|c|}{$\begin{array}{l}\text { ORDINATEUR (clé / } \\
\text { réalisation de la tâche) }\end{array}$} & 10 & $\begin{array}{l}1,8 \\
(2)\end{array}$ & 1,4 & $\begin{array}{l}3,8 \\
(2,4)\end{array}$ & 10,2 \\
\hline
\end{tabular}

\section{Tableau 5 - Nombre moyen, écart-type et proportion d'échanges par catégorie selon le mode de communication (étude 1)}

Nous observons que, dans les deux conditions, la majorité des échanges entre étudiants concerne la carte (mise en forme, relations et concepts : $58 \%$ des échanges en face à face et 50,4\% des échanges pour les utilisateurs du chat). Dans les deux cas, la catégorie comptabilisant le plus d'interventions est la catégorie traitant des concepts $(29,7 \%$ en face à face et $27,9 \%$ à distance). De la même manière, les proportions sont identiques pour les autres catégories : la différence de mode de communication n'entraîne pas plus d'interventions sur l'utilisation du logiciel (8,8 \% en face à face et $7,1 \%$ à distance), ni sur la tâche ellemême (organisation et définition : 16,2\% pour les dyades communiquant en face à face et 9,3\% pour ceux à distance), ni sur le nombre de références au texte (8,2\% en face à face versus $3,5 \%$ à distance).

La seule catégorie montrant une liaison significative du nombre d'échanges avec le mode de communication est la dernière catégorie, qui renvoie à l'utilisation de l'ordinateur pour réaliser la tâche, avec des proportions de $1,4 \%$ d'échanges dans la situation de communication en face à face et de $10,2 \%$ pour la communication à distance : $\left.\mathrm{X}^{2}(\mathrm{dd} 11, \underline{\mathrm{N}}=11)=7,02 ; \mathrm{p}<.001\right)$. En effet, dans la majorité des dyades interagissant en face à face, une seule des deux personnes utilise le logiciel et réalise la carte en 
prenant en compte l'avis du partenaire. Ainsi, les interventions concernant l'utilisation du logiciel sont du type "tu veux le faire à l'ordinateur ?". Dans un seul binôme, nous observons un changement de l'utilisateur de l'ordinateur (dyade 10), et ceci n'a eu lieu qu'une seule fois. Dans la condition "communication à distance", les interlocuteurs doivent demander la clé pour pouvoir manipuler l'espace de travail. Nous observons en moyenne 9,8 changements de clé, avec toutefois une variabilité inter dyades importante (écart-type $=6,9$, avec un binôme présentant seulement deux passages de clé et un autre en comptabilisant 22).

Ainsi, les objets des échanges entre étudiants sont sensiblement les mêmes dans les deux conditions de collaboration comparées (face à face et à distance), dans des proportions équivalentes (carte, tâche, utilisation du logiciel ou références au texte); seule l'utilisation de l'ordinateur engendre des interactions portant spécifiquement sur le passage de la clé dans la condition à distance.

\subsubsection{Comparaisons entre étudiants universitaires et collégiens communiquant à distance}

Comme pour la première étude, nous présentons les données des analyses quantitative et qualitative des échanges enregistrés ${ }^{\underline{6}}$. L'analyse des cartes conceptuelles produites par les collégiens n'est pas présentée, car la comparaison avec les cartes produites par les étudiants universitaires a peu de sens compte tenu des différences entre les textes qui leur étaient soumis.

\subsubsection{Analyse quantitative}

Les mêmes critères que dans l'étude précédente ont été retenus pour l'analyse quantitative des données, à savoir la durée de l'interaction, le nombre d'interventions et d'échanges, le nombre d'actions opérées sur le modèle ainsi que la structure des échanges (tableaux 6 et 7). Les données concernant les collégiens sont mises en regard de celles concernant les étudiants, uniquement à titre indicatif car elles ne sont pas strictement comparables, compte tenu des différences existant entre les textes considérés et des contextes éducatifs (les séquences de travail sont habituellement plus longues à l'université qu'au collège).

\begin{tabular}{|c|c|c|c|}
\hline & & Collégiens & $\begin{array}{l}\text { Étudiants } \\
\text { universitaire } \\
\text { S }\end{array}$ \\
\hline \multirow{2}{*}{$\begin{array}{l}\text { durée de l'interaction } \\
\text { (en minutes) }\end{array}$} & Moyenne & 29,9 & 80,9 \\
\hline & écart type & 7,1 & 10 \\
\hline \multirow{2}{*}{$\begin{array}{ll}\text { nombre } & \text { total } \\
\text { d'interventions }\end{array}$} & Moyenne & 34,9 & 81,8 \\
\hline & écart type & 16,4 & 42 \\
\hline \multirow{2}{*}{ nombre d'échanges } & Moyenne & 14,4 & 37,7 \\
\hline & écart type & 5,3 & 14,3 \\
\hline \multirow{2}{*}{$\begin{array}{l}\text { nombre d'actions } \\
\text { opérées } \\
\text { sur le modèle }\end{array}$} & Moyenne & 268,2 & 691,2 \\
\hline & écart type & 89 & 130 \\
\hline
\end{tabular}

Tableau 6 - Comparaison des deux populations selon les différents critères d'analyse 


\begin{tabular}{|c|c|c|c|c|}
\hline & \multicolumn{2}{|l|}{ Collégiens } & \multicolumn{2}{|c|}{$\begin{array}{l}\text { Étudiants } \\
\text { universitaires }\end{array}$} \\
\hline & $\begin{array}{l}\text { Moyenne } \\
\text { (écart- } \\
\text { type) }\end{array}$ & $\begin{array}{l}\text { Proportion } \\
\text { (en \%) }\end{array}$ & $\begin{array}{l}\text { Moyenne } \\
\text { (écart- } \\
\text { type) }\end{array}$ & $\begin{array}{l}\text { Proportion } \\
\text { (en \%) }\end{array}$ \\
\hline $\begin{array}{l}\text { Echanges } \\
\text { tronqués }\end{array}$ & $\begin{array}{l}6,2 \\
(2,8)\end{array}$ & 43,5 & $\begin{array}{l}16,7 \\
(8,3)\end{array}$ & 44,2 \\
\hline $\begin{array}{l}\text { Echanges } \\
\text { restreints }\end{array}$ & $\begin{array}{l}2,7 \\
(2)\end{array}$ & 19,1 & $\begin{array}{l}11,5 \\
(1,4)\end{array}$ & 30,5 \\
\hline $\begin{array}{l}\text { Echanges } \\
\text { étendus }\end{array}$ & $\begin{array}{l}5,4 \\
(3)\end{array}$ & 37,4 & $\begin{array}{l}9,5 \\
(6,8)\end{array}$ & 25,2 \\
\hline
\end{tabular}

\section{Tableau 7 - Nombre moyen, écart-type et proportion d'échanges tronqués, restreints et étendus pour chaque population}

Chez les collégiens comme chez les étudiants universitaires, les échanges sont majoritairement tronqués : l'intervention verbale de l'un n'entraîne pas de réponse verbale de la part de l'autre interlocuteur. La fréquence de ce mode de communication associée à l'absence de rupture communicationnelle apparente laisse supposer que les interlocuteurs mettent en relation le dire et le faire, ce que permet le partage de l'espace de travail. Les échanges étendus sont plus nombreux chez les collégiens et les échanges restreints sont plus nombreux chez les étudiants universitaires. Toutefois, ces différences n'atteignent pas la significativité $\left(\mathrm{X}^{2}=3,08\right.$ pour les échanges restreints et $\mathrm{X}^{2}=3,34$ pour les échanges étendus).

\subsubsection{Analyse qualitative : objet des échanges}

Le contenu des échanges entre adolescents a fait l'objet de la même analyse que pour les adultes. Les données de la comparaison sont indiquées dans le tableau 8.

\begin{tabular}{|c|c|c|c|c|c|c|}
\hline & & & \multicolumn{2}{|c|}{ Collégiens } & \multicolumn{2}{|c|}{$\begin{array}{l}\text { Étudiants } \\
\text { universitaires }\end{array}$} \\
\hline & & & $\begin{array}{l}\text { Moyenne } \\
\text { (écart- } \\
\text { type) }\end{array}$ & $\begin{array}{l}\text { Proportion } \\
\text { (en \%) }\end{array}$ & $\begin{array}{l}\text { Moyenne } \\
\text { (écart- } \\
\text { type) }\end{array}$ & $\begin{array}{l}\text { Proportion } \\
\text { (en \%) }\end{array}$ \\
\hline \multirow{3}{*}{ CARTE } & $\begin{array}{l}\text { mise en } \\
\text { forme }\end{array}$ & 1 & $\begin{array}{l}3,1 \\
(1,8)\end{array}$ & 21,7 & $\begin{array}{l}3,5 \\
(2,8)\end{array}$ & 9,3 \\
\hline & liens & 2 & $\begin{array}{l}1,1 \\
(1,1)\end{array}$ & 7,8 & $\begin{array}{l}5 \\
(4,1)\end{array}$ & 13,3 \\
\hline & concept & 3 & $\begin{array}{l}2 \\
(1,4)\end{array}$ & 13,9 & $\begin{array}{l}10,5 \\
(4,5)\end{array}$ & 27,9 \\
\hline LOGICIEL & utilisation & 4 & $\begin{array}{l}0,1 \\
(0,3)\end{array}$ & 0,9 & $\begin{array}{l}2,7 \\
(2)\end{array}$ & 7,1 \\
\hline \multirow{2}{*}{ TACHE } & organisation & 5 & $\begin{array}{l}3,1 \\
(2,8)\end{array}$ & 21,7 & $\begin{array}{l}3,5 \\
(0,8)\end{array}$ & 9,3 \\
\hline & définition & 6 & $\begin{array}{l}0 \\
(0)\end{array}$ & 0 & $\begin{array}{l}0 \\
(0)\end{array}$ & 0 \\
\hline
\end{tabular}




\begin{tabular}{|l|l|l|l|l|l|l|}
\hline TEXTE & $\begin{array}{l}\text { Référence/ } \\
\text { citation }\end{array}$ & 7 & $\begin{array}{l}0,2 \\
(0,7)\end{array}$ & $\mathbf{1 , 7}$ & $\begin{array}{l}1,3 \\
(2,4)\end{array}$ & $\mathbf{3 , 5}$ \\
\hline \hline $\begin{array}{l}\text { RELATIONS } \\
\text { SOCIALES }\end{array}$ & 8 & $\begin{array}{l}0,9 \\
(0,8)\end{array}$ & $\mathbf{6 , 1}$ & $\begin{array}{l}1,8 \\
(0,7)\end{array}$ & $\mathbf{4 , 9}$ \\
\hline AUTRE & 9 & $\begin{array}{l}1,5 \\
(1,2)\end{array}$ & $\mathbf{1 0 , 4}$ & $\begin{array}{l}5,5 \\
(5,1)\end{array}$ & $\mathbf{1 4 , 6}$ \\
\hline $\begin{array}{l}\text { ORDINATEUR (clé / } \\
\text { réalisation de la tâche) }\end{array}$ & 10 & $\begin{array}{l}2,2 \\
(1,6)\end{array}$ & $\mathbf{1 5 , 6}$ & $\begin{array}{l}3,8 \\
(2,4)\end{array}$ & $\mathbf{1 0 , 2}$ \\
\hline
\end{tabular}

\section{Tableau 8 : Nombre moyen, écart-type et proportion d'échanges par catégorie selon l'âge pour chaque population}

Pour les collégiens comme pour les étudiants universitaires, la majorité des échanges concerne la carte elle-même (respectivement $43,5 \%$ et $50,4 \%$ pour l'ensemble des trois catégories de la grille se référant à la carte). Toutefois, la majorité des échanges des collégiens a trait à la mise en forme de la carte $(21,7 \%$ contre $9,3 \%$ chez les adultes ; $\left.\mathrm{X}^{2}(\mathrm{ddl} 1, \mathrm{~N}=14)=5,91 ; \mathrm{p}<.005\right)$. Les échanges portant sur les relations entre les concepts sont équivalents chez nos deux populations (respectivement 7,8\% et 13,3\% chez les collégiens et les étudiants universitaires respectivement) et les étudiants universitaires privilégient les interactions concernant les concepts $(27,9 \%$ chez les collégiens versus $13,9 \%$; chez les étudiants universitaires ; $\left.\mathrm{X}^{2}(\mathrm{ddl} 1, \underline{\mathrm{N}}=14)=5,90 ; \mathrm{p}<.005\right)$. Ainsi, si la majorité des échanges concerne directement la carte pour nos deux populations, les collégiens se focalisent principalement sur sa mise en forme alors que les étudiants universitaires traitent davantage des concepts.

Concernant l'utilisation du logiciel, peu d'échanges y ont trait, et beaucoup moins chez les collégiens $\left(0,9 \%\right.$ des échanges versus $7,1 \%$ chez les adultes ; $\left.X^{2}(d d 11, \underline{N}=14)=5,05 ; p<.005\right)$, ce qui indique que son utilisation ne leur pose pas problème. Une grande partie des échanges entre collégiens concerne l'organisation de la tâche $(21,7 \%$ des échanges, ce qui représente la plus grande proportion d'échanges avec la catégorie "mise en forme"), alors que cette catégorie ne représente que 9,3\% des échanges entre les étudiants universitaires $\left(\mathrm{X}^{2}(\mathrm{ddl} 1, \underline{\mathrm{N}}=14)=5,91 ; \mathrm{p}<.005\right)$. En d'autres termes, les collégiens se focalisent sur l'organisation de la tâche et sur la mise en forme de celle-ci, alors que les étudiants universitaires s'attachent principalement à discuter des concepts devant figurer sur la carte.

Les autres catégories sont représentées de façon équivalente chez nos deux populations (référence au texte, relations sociales, clé, autre).

\section{Discussion}

Les résultats essentiels qui se dégagent de cette étude sont récapitulés ci-après.

Dans la première étude qui a impliqué des étudiants universitaires, la qualité des cartes conceptuelles produites est comparable dans les deux situations de communication (en face à face et à distance), du point de vue du nombre de concepts ou du nombre de relations établies entre les concepts.

En revanche, le mode de communication a un impact sur les échanges au plan de leur structure. En effet, la communication en face à face favorise les échanges étendus alors que la communication à distance se traduit par des échanges tronqués. De plus, les nombres d'interventions et d'échanges sont nettement plus nombreux chez les sujets communiquant en face à face. Par ailleurs, les dyades qui communiquent à distance mettent plus de temps à réaliser le travail que ceux qui communiquent en face à face (près d'un quart d'heure de plus), et leur nombre moyen d'actions opérées sur le modèle est nettement supérieur. Il semble, selon nous, que cet ensemble d'observations reflète deux modes de fonctionnement tout à fait distincts. En effet, les sujets qui travaillent en face à face échangent leurs points de vue dans un premier 
temps, puis réalisent la carte à l'aide du logiciel dans un second temps. On observe systématiquement une mise en accord préalable à l'action sur le modèle. Lorsque les sujets communiquent à distance, ils utilisent directement l'ordinateur qui représente leur seul moyen de communication. Ainsi, ce sont les actions qui deviennent les plus importantes, et non l'interaction dialogique. Ce constat explique selon nous les différences entre le type d'échanges observés: des échanges étendus chez les sujets communiquant en face à face, qui privilégient l'interaction verbale par rapport aux actions sur le modèle, et des échanges tronqués chez les sujets communiquant à distance, pour lesquels l'action est davantage utilisée. Ainsi, pour les sujets communiquant via le chat, l'interaction est essentiellement centrée sur l'action : l'envoi d'un message n'est pas systématiquement suivi d'une réponse écrite mais plutôt d'un ajustement de l'action en cours, ce qui explique que la lecture de l'ensemble des messages écrits des partenaires d'une dyade forme un dialogue tronqué que comblent des actions sur le modèle.

Le deuxième résultat important de cette étude concerne le contenu des échanges. En effet, nous observons que l'objet des échanges est comparable dans les deux conditions de communication: les sujets s'occupent principalement de la carte (et notamment des concepts et des relations entre les concepts) dans les deux cas. Sur les dix catégories que compte la grille d'analyse, neuf sont représentées dans des proportions équivalentes dans les deux conditions de communication. Seule la catégorie inhérente à l'utilisation de l'ordinateur permet de repérer des différences entre les deux groupes, cela s'expliquant par la tâche elle-même puisque seule l'utilisation de l'ordinateur permet la communication dans un des deux groupes. Notons toutefois que la communication à distance semble engendrer un meilleur partage de la tâche : si pour les dyades communiquant en face à face, c'est essentiellement une seule des deux personnes qui manipule le logiciel, pour ceux communiquant à distance, les interactants se répartissent le travail. On comptabilise en effet en moyenne 9,38 changements de main de la clé dont la possession rend possible les actions sur le modèle. Cela rejoint le point précédent : l'interaction passe par l'action sur le modèle davantage qu'au moyen d'échanges verbaux écrits.

Notre seconde étude est une réplication de la modalité "communication à distance" chez une population adolescente (collégiens âgés de 14 ans en moyenne) ayant une moindre maîtrise de l'écrit que la population étudiante impliquée dans la première étude. Il s'agissait d'examiner si cela pouvait avoir un impact sur la communication entre pairs. Aussi avons-nous procédé à une comparaison de la structure et du contenu des échanges entre les collégiens avec ce qui a été observé chez les étudiants universitaires dans la même condition. Là aussi, deux résultats importants se dégagent.

D'une part, la structure des échanges des collégiens est comparable à celle des étudiants universitaires. Nous observons en effet une majorité d'échanges tronqués (représentant quasiment la moitié des échanges chez nos deux populations). Ainsi, c'est également l'action qui est privilégiée par les collégiens communiquant par écrit.

En revanche, l'analyse de l'objet des échanges révèle que les collégiens échangent principalement au sujet de la mise en forme de la carte et de l'organisation du travail. Ces deux catégories représentent environ la moitié des échanges de ces adolescents (43,44\% des échanges précisément). Selon nous, le fait que la majorité des échanges des adolescents concernent les catégories "mise en forme" de la carte d'une part et "organisation" de la tâche, d'autre part, reflète l'absence de collaboration réelle pour cette population. En effet, la majorité des dyades semble avoir adopté la stratégie suivante : choix d'une des deux cartes individuelles puis réplication de la carte choisie avec orientation des messages sur la mise en forme. Ainsi, une grande partie des échanges vise d'abord à déterminer quelle carte sera répliquée, donnant lieu à des échanges du type : "On prend la tienne ou la mienne ${ }^{7}$ ? "; "tu veux que l'on prenne

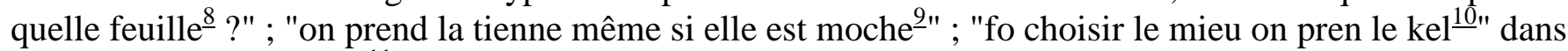
le début de l'interaction ${ }^{11}$. Ensuite, c'est sur la mise en forme de la carte que s'orientent principalement les dialogues, avec des interventions de type : "oué ça va mais petetre ke le titre est pa assez gros $\underline{12}$ "; "c po droit / decal" ; "mé fabbrication é reproduciton a la mém oteur / mé synthétiqu o dessu de réaktion ${ }^{13}$ ". Ainsi, nous n'observons pas de confrontation de point de vue sur le choix des concepts ou sur les liaisons 
à établir entre les concepts, et de façon générale de mise en commun des deux cartes individuelles.

Tout se passe comme si les deux populations avaient investi le logiciel de différentes manières : pour les étudiants, il semble être un outil permettant de travailler une lecture conceptuelle du texte, alors que pour les collégiens il est surtout un outil graphique. Ces différences renvoient sans doute à des usages très différents des ordinateurs par ces deux populations.

Pour conclure, il semble que l'utilisation de l'écrit engendre des fonctionnements différents, davantage centrés sur l'action et ce, chez l'adulte comme chez l'adolescent. Il semble toutefois nécessaire de répliquer ce type de protocole afin d'étayer davantage nos conclusions, notamment en répliquant la modalité "face à face" auprès de la population adolescente.

Notons par ailleurs que, lors des différentes études menées par les équipes du consortium ModellingSpace, les élèves (d'écoles élémentaires et de collège) ont tous montré une grande motivation et une grande aisance à manipuler le logiciel, qui permet d'envisager de multiples activités. Plusieurs domaines ont été abordés dans ces études : les sciences-physiques (Smyrnaiou et Weil-Barais, 2003); (Smyrnaiou et Weil-Barais, 2005), la résolution de problèmes (Avouris et al., 2003), la chimie (Kommatas et al., 2004) ou encore la géographie (Strebelle et Depover, 2003). Il s'avère que le logiciel offre de nombreuses possibilités d'études grâce aux outils d'analyse dont il est doté.

L'évaluation que nous avons conduite a procédé principalement d'une approche comparative entre des conditions d'utilisation du logiciel pour des populations très contrastées, en tentant autant que faire se peut de contrôler le contexte relationnel entre les participants dont on sait par ailleurs qu'il intervient sur la qualité des interactions. Nous avons pris soin également de placer les participants dans des conditions de travail favorables en regard des connaissances acquises sur le travail en groupe. En effet, comme le remarquent un certain nombre d'auteurs, toute situation de collaboration n'est pas nécessairement favorable et appréciée des élèves et des étudiants (Arnaud, 2003). Par ailleurs, la tâche a été choisie de telle sorte que le logiciel assure les fonctions pour lesquelles il a été conçu (modélisation, communication, métacognition). Ceci nous semble constituer des conditions nécessaires pour procéder à une évaluation des logiciels éducatifs. De manière générale, on peut supposer en effet que leur impact en termes d'apprentissage et de développement des compétences dépend d'une meilleure connaissance des conditions qui en permettent un usage optimal. De notre point de vue, c'est par des études suffisamment précises concernant la manière dont les élèves les utilisent dans des contextes bien spécifiés qu'on pourra avancer dans cette connaissance. C'est pourquoi il nous semble nécessaire de doter les logiciels éducatifs d'outils permettant le recueil et le traitement de données utiles pour conduire des évaluations pas trop coûteuses comme celle que nous avons présentée dans cet article, ce qui permet de les reproduire facilement dans différents contextes éducatifs.

\section{Références}

ARNAUD, M. (2003). Les limites actuelles de l'apprentissage collaboratif en ligne. Sciences et Technologies de l'Information et de la Communication pour l'éducation et la Formation, 10, mis en ligne le 15-11-2003, http://sticef.org, ISSN : p. 1764-7223.

AVOURIS, N., DIMITRACOPOULOU, A., KOMIS, V. (2003). On analysis of collaborative problem solving : an object oriented approach. Computers in Human Behavior, 19 (2), p.147-167.

BARBIER, M.L., JAL-JAL, F., PIOLAT, A., AGOSTINELLI, S. (1993). Hypertexte et réseau : une expérience sur la rédaction interactive de textes. Enseignement Public et Informatique, 72, p. 89-95.

BAUDRIT, A. (1997). Apprendre à deux. Etudes psychosociales de situations dyadiques. Paris : PUF.

CARBONES I FLETA, A.M., CUISINIER, F. (2003). Amitié et cognition : une démarche d'analyse des échanges entre enfants confrontés à des tâches scolaires. Bulletin de Psychologie, 56 (4), p. 615-624. 
CHABROL, C., BROMBERG, M. (1999). Préalables à une classification des actes de parole. Psychologie Française, 44 (4), p. 291-306.

CRINON, J. (1993). Lire pour écrire, écrire pour être lu. Cahiers Pédagogiques, 314/315, p. 60-63.

CRINON, J., MANGENOT, F., GEORGET, P. (2002). Communication écrite, collaboration et apprentissages. In D. LEGROS et J. CRINON (Éds.), Psychologie des apprentissages et multimédia (p. 63-83). Paris: Armand Colin.

DA SILVA, E. (2003). Les interactions de co-résolution de problèmes entre pairs : aspects sociaux, aspects cognitifs et enjeux pédagogiques. Bulletin de Psychologie, 56 (4), p. 609-614.

DIMITRACOPOULOU, A. (2003) Modelling Activities in School with a collaborative technology based learning environment. Scenarios of use in secondary education. In P. MASSOURAS. (Ed.), Information, technologies and education. CCS, Cyprus.

DOISE, W. (1993). Logiques sociales dans le raisonnement. Neuchâtel, Delachaux et Niestlé.

GEORGE, S. (2003). Analyse automatique de conversations textuelles synchrones d'apprenants pour la détermination des comportements sociaux. Sciences et Technologies de l'Information et de la

Communication pour l'éducation et la Formation, 10, mis en ligne le 15-11-2003, http://sticef.org, ISSN : p. $1764-7223$.

GILLY, M., FRAISSE, J., ROUX, J.P. (1988). Résolution de problèmes en dyades et progrès cognitifs chez des enfants de 11 à 13 ans. In A.N. PERRET-CLERMONT \& M. NICOLET (Eds). Interagir et connaître : enjeux et régulations sociales dans le développement cognitif (pp. 73-92). Cousset (Fribourg) : Delval.

GILLY, M. (1993). Psychologie sociale des constructions cognitives : perspectives européennes. Bulletin de Psychologie, 412, p. 671-683.

GOLDMAN, S. (1996). Reading, writing, and learning in hypermedia environments. In H. van Oostendorp, S. De Mul (Eds.), Cognitive aspects of electronic texts processing (p. 7-42). Norwood, NJ: Ablex.

HAWISHER, G.E., SELFE, C.S. (1999). Reflections on research in computers and composition studies at the century's end. In J. Hancock (Ed.), Teaching Literacy Using Information Technology (p. 31-47).

Newark, Delaware: International Reading Association.

JEHNG, J.C.J. (1997). The psycho-social processes and cognitive effects of peer-based collaborative interactions with computers. Journal of Educational Computing Research, 17(1), p.19-46.

JOHNSON, D.W., JOHNSON, R.T. (1998). Cooperative learning and social interdependence theory. En ligne: www.clcrc.com/pages/SIT.html

JONASSEN, D.H., REEVES, T. \& HONG, N. (1998). Concept mapping as cognitive learning and assessment tools. Journal of Interactive Learning Research, 8, p. 289-308.

KERBRAT-ORECCHIONI, C. (1990). Les interactions verbales. Paris : Armand Colin.

KOMIS, V., DIMITRACOPOULOU, A., POLITIS, P., AVOURIS, N. (2001). Expérimentation sur l'utilisation d'un logiciel de modélisation par petits groupes d'élèves. Sciences et Techniques Educatives, 8 (1-2), p. 75-86.

KOMMATAS, N., FORTOUNI, T., FRAGKAKI, M, PASSALIS, N., DIMITRACOPOULOU, A. (2004). Combining experiments in chemistry with technology based cognitive tools, for students of primary schools. In M. Grigoriadou (Ed.). Proceedings of the 4th Hellecnic Congress with International Participation, ICTs in Education (p. 25-34). Athens: New technologies editions. 
LEHTINEN, E., HAKKARAINEN, K., LIPPONEN L., RAHIKAINEM, M., MUUKKONEN, H. (1998). Computer-Supported Collaborative Learning: A Review. CL-Net Project. En ligne: http://suvi.kas.utu.fi/papers/clnet/clnetreport.html.

MC ALEESE, R. (1998). The knowledge arena as an extension to the concept map: reflection in action. Interactive Learning environments, 6(3), p. 251-272.

MARGARITIS, M., KOMIS, V., AVOURIS, N., DIMITRACOPOULOU, A. (2003). Real-time collaborative modelling over low-bandwidth networks. In B. WASSON, R. BAGGETUN, U. HOPE \& S. LUDVIGSEN (Eds), Proceedings of the Computer Support for Collaborative Learning, June 14-18 (p. 138-140). Bergen: University of Bergen Editions.

NOVAK, J.D., GOWIN, D.B. (1984). Learning how to learn. Cambridge, London: Cambridge University Press.

PALINSCAR, A. S. (1998). Social constructivist perspectives on teaching and learning. Annual Review of Psychology, 49, p. 345-375.

PLéTY, R. (1996). L’apprentissage coopérant. Lyon : PUF.

PONTECORVO, C. (1988). Interactions socio-cognitives et acquisition des connaissances en situation scolaire : contextes théoriques, bilan et perspectives. European Journal of Psychology of Education. Numéro spécial hors-série : Le fonctionnement de l'enfant à l'école, p. 139-149.

PUGACH, H., JOHNSON, L.J. (1995). Collaborative practitioners and collaborative schools. Denver: Love Publishing Company.

SCARDAMALIA, M., BEREITER, C. (1986). Research on written composition. In M. C. WITTROCK (Ed.), Handbook of research on teaching (p. 778-803). New-York: McMillan.

SMYRNAIOU, Z., WEIL-BARAIS, A. (2003). Evaluation cognitive d'un logiciel éducatif destiné à l'éducation scientifique. In C. CONSTANTINOU (Ed). Computer Based Learning in Science, Proceeding of $6^{\text {th }}$ International Conference, University of Cyprus, Nicosia, 5-10 juillet.

SMYRNAIOU, Z., WEIL-BARAIS, A. (2005). Evaluation cognitive d'un logiciel de modélisation auprès d'élèves de collège. Didaskalia, 27, p. 133-149.

STACEY, E. (1998). Study of the enhancement of learning through group interaction by computer mediated communication. Thèse de doctorat, Melbourne Monash University.

TRIBOLLET, B., LANGLOIS, F., GREA, J. (1997). Emploi des cartes conceptuelles au lycée et en formation des maîtres. Rapport à la Région de l'action $n^{\circ}$ HO 3914-9424. Ed SUFRESP - UCBL, Lyon.

STREBELLE, A., DEPOVER, C. (2003). Implication d'une communauté de praticiens innovateurs dans le design et l'implantation d'un environnement collaboratif de modélisation. Actes du $2^{\text {ème }}$ colloque de Guéret "Les communautés virtuelles éducatives", 4-6 juin.

VYGOTSKI, L. (1934/1978). Pensée et Langage. Paris : La Dispute.

WEIL-BARAIS, A. (2004). Les apprentissages scolaires. Rosny sous Bois : Editions Bréal.

YAVERBAUM, G.J., OCKER, R.J. (1998). Problem solving in the virtual classroom : a study of students perceptions related to collaborative learning techniques. WebNet 98 World Conference of the WWW, Internet and Intranet proceedings, Orlando, FL, November 7-12, 1998. (ERIC Document No. ED 427 750)..

ZAMMUNER, V.L. (1995). Individual and cooperative computer-writing and revising: who gets the best results? Learning and Instruction, 5 (2), p. 101-124. 


\section{Annexe 1: Texte proposé aux adolescents HISTOIRE DE QUELQUES SUBSTANCES SYNTHÉTIQUES}

Une substance synthétique est une substance fabriquée par l'homme, par opposition à une substance naturelle, c'est-à-dire que l'on trouve directement dans la nature, comme par exemple de l'eau, de l'air, du pétrole, du sel, du sable, le jus d'un fruit etc.

Une matière synthétique est préparée en faisant réagir ensemble - on appelle cela une réaction chimique des matières, qui elles-mêmes peuvent être soit naturelles soit déjà synthétiques. La technique la plus ancienne pour déclencher une réaction chimique est de chauffer les matières en présence. Comme substance synthétique, on pense bien sûr souvent aux matières plastiques, aux médicaments... mais le pain que nous mangeons tous les jours est aussi une substance synthétique !

Jusqu'au XVIII $^{\mathrm{e}}$ siècle, les hommes synthétisaient les substances au petit bonheur la chance. Ils essayaient de faire réagir ensemble différentes matières et regardaient si le produit ainsi obtenu présentait un quelconque intérêt. C'est ainsi qu'ont été fabriqués, un peu par chance, le fer, les premiers savons, le verre, des pigments...

Puis, à partir du début du XIX ${ }^{\mathrm{e}}$ siècle, apparaît l'analyse chimique, ensemble de méthodes qui permettent d'identifier quels sont les atomes présents dans telle ou telle substance. Dès lors, les chimistes ne travaillent plus en aveugle: ils vont s'efforcer de copier la nature, en synthétisant notamment des molécules qui existent déjà dans les plantes. C'est ainsi qu'ils copient des molécules de colorants, de médicaments, de parfums etc. Comme ces produits sont fabriqués en grande quantité dans les usines, ils coûtent beaucoup moins cher que les substances naturelles dont ils sont la copie.

En ce début de $\mathrm{XXI}^{\mathrm{e}}$ siècle, on peut dire que l'homme est maintenant capable de copier la plupart des substances naturelles (mais pas toutes cependant). Il est également capable de synthétiser des produits qui n'existent pas dans la nature, comme de nombreuses matières plastiques par exemple.

(306 mots, 30 lignes) 


\section{Annexe 2 : Exemple de carte conceptuelle et de dialogue du chat d'adultes}

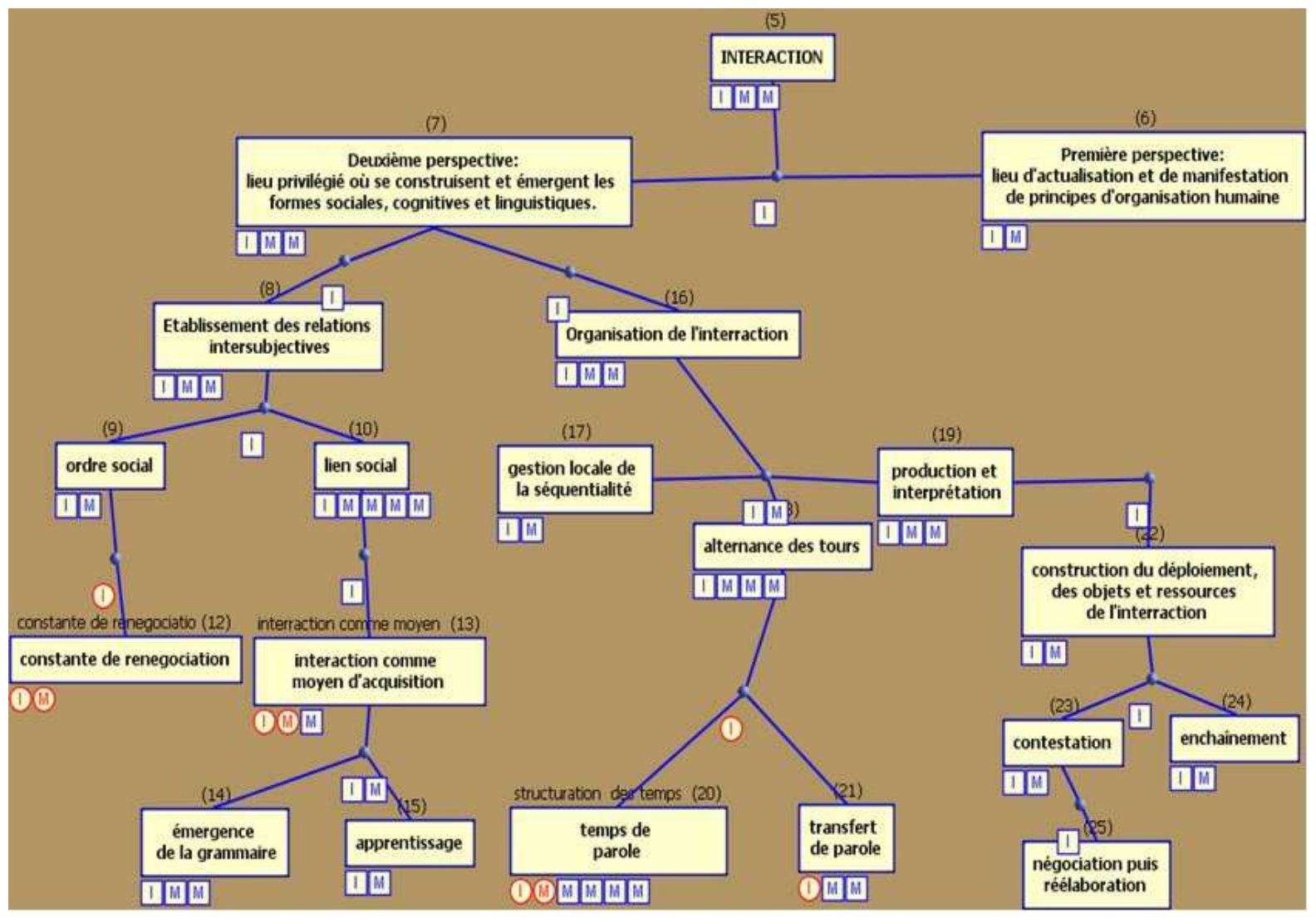

[S2] bonjour

[ S17] on va bien rigoler dis donc!!! on met début travail $15 \mathrm{~h}$ ?

[ S2] oui. on commence par regarder ce qu'on a fait? ou on y va direct?

[ S17] Là je suis en train de regarder les points communs! on se fait signe des que ça c'est fait je pense.

[S2] OK

[ S17] bon, je pense qu'on a les mêmes idées! Moi j'en ai trop mis je pense. il y a seulement un petit lien qui nous différentie: c'est pour "l'action prospective et rétrospective", moi je l'ai relié avec "alternance tours de parole" et toi avec "organisée par la gestion locale de la séquentialité", mais je sais pas si c'est très grave. est ce qu'on relie le texte?

[ S2] On a fait à peu près pareil mais organisé un peu différamment, je suis d'accord. POur l'action prospective et rétrospective je l'ai relié à la gestion locale de la séquentialité car c'est dans le meme paragraphe. mais si tu regarde la gestion locale de la séquentialité est liée à l'alternance des tours de parole donc c'est pareil que toi. Ta carte est assez compliquée quand meme! En fait il faudrait essayer de regrouper plus de choses.

[ S17] c'est à dire? Prendre + d'elements du texte?en fait je pense que le tienne est quand même vachement bien résumée! elle est claire!(pas comme moi certes!!)on peut essayer de voire ce qu'on oublié dans le texte?!

[ S2] Moi j'ai oublié certain détails mais la tienne est complète. je pense qu'il faudrait reprendre les éléments de ta carte et les organiser avec l'aide de la mienne. Car si on commence à relire le texte on va plus s'en sortir. 
Revue sticef.org

\section{dialogue du chat d'adolescents}

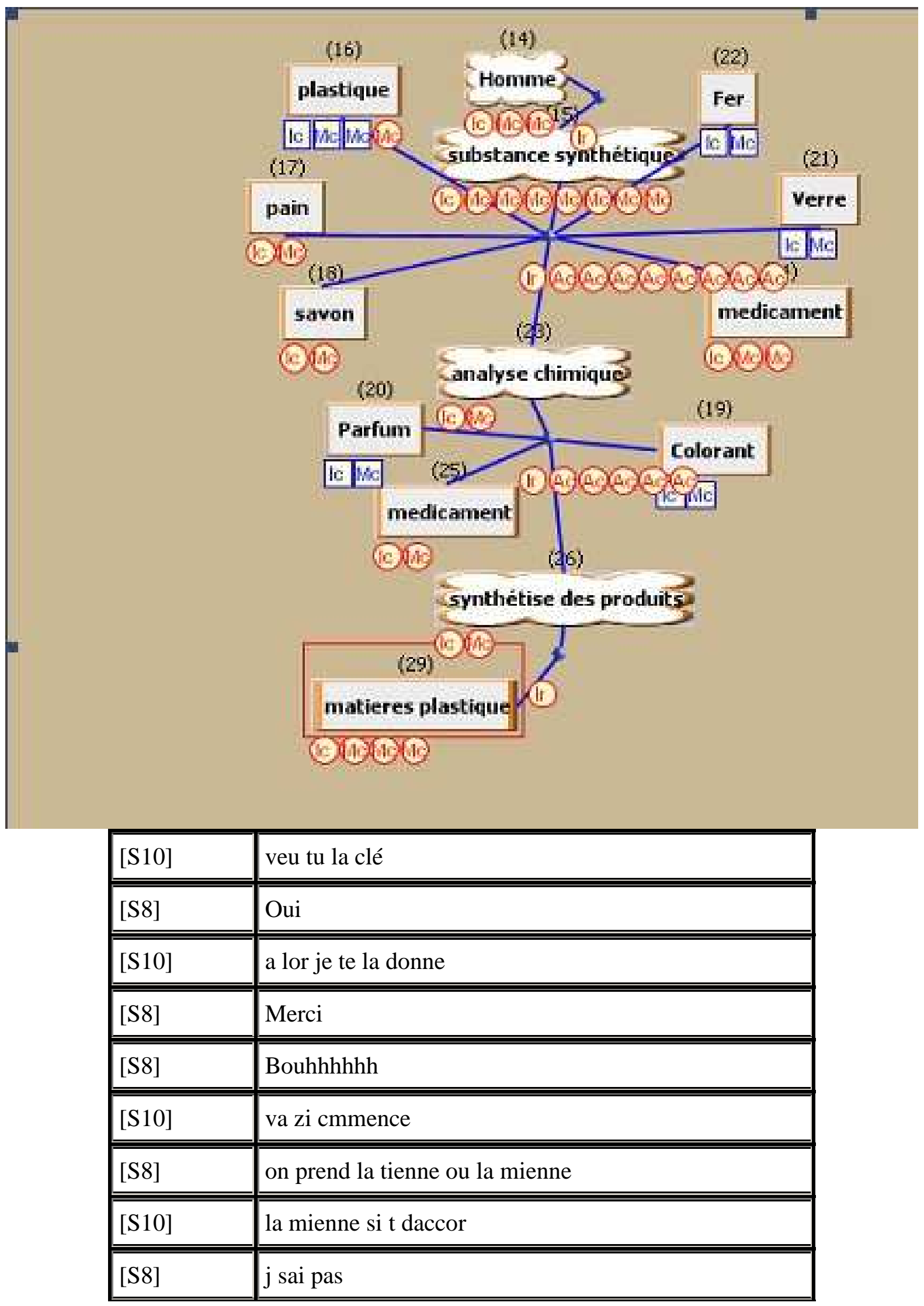




\begin{tabular}{|c|c|}
\hline [S8] & ???????????? \\
\hline$[\mathrm{S} 10]$ & tan pi on pren la mienne \\
\hline [S8] & C EST PYUOR TOI \\
\hline [S8] & C EST POUR TOI \\
\hline [S8] & NON AU millieu \\
\hline [S8] & met un lien \\
\hline [S8] & Bien \\
\hline [S8] & je vai $\mathrm{t}$ aider \\
\hline [S8] & passe moi la clé pour les liens \\
\hline$[\mathrm{S} 10]$ & noubli pa on fait le mien \\
\hline [S8] & ca te va \\
\hline
\end{tabular}

${ }^{1}$ Les universités de Aegean et de Patras en Grèce, de Mons-Hainaut en Belgique, d'Angers en France ainsi que la Nouvelle Université de Lisbonne au Portugal et l'entreprise espagnole SchlumbergerSema ont pris part à ce projet clôturé en mars 2004.

$\stackrel{2}{2}$ Houdé O., Kayser, D., Koenig, O., Proust, J., Rastier, F. (1998). Vocabulaire des sciences cognitives. Paris: PUF.

$\underline{3}$ Voir annexe 1 .

${ }^{4}$ Un exemple d'une carte produite et de dialogue du chat est fourni en annexe 2.

5 Dans ce type d'échange, les interventions verbales sont généralement suivies d'une action de l'interlocuteur. Cette action ne fait pas l'objet de feedback. Ce genre d'échanges, s'il perdure lors d'une interaction, est l'expression d'accords tacites entre les interactants. En face à face, ce type de fonctionnement suscite généralement une rupture entre les interactants.

${ }^{6}$ Un exemple de production de carte et de dialogue du chat de collégiens est fourni en annexe 3.

7 dyade 5 ; échange 3

$\underline{8}$ dyade 2 ; échange 1

9 dyade 6 ; échange 3

10 dyade 4 ; échange 2

11 Notons également que les échanges des collégiens se caractérisent par le style rédactionnel habituellement réservé à l'envoi de «textos », ce qui est nettement moins généralement observé chez les adultes.

12 dyade 8 , échange 12

13 dyade 4 ; échange 5 


\section{- A propos des auteurs}

Aurélie Lainé est maître de conférence à l'IUFM des Pays de la Loire et membre du CREN (Centre de Recherches en Education de Nantes, Université de Nantes). Elle était auparavant membre de l'équipe «Cognition et Développement » du laboratoire de psychologie «Processus de pensée » de l'université d'Angers, où elle a contribué à l'évaluation d'un logiciel éducatif expérimental dans le cadre du programme de recherche européen ModellingSpace sous la responsabilité du Pr A. Weil-Barais.

Adresse : IUFM des Pays de la Loire, site d'Angers ; 7 rue Dacier 49100 Angers

Courriel : aurelie.laine@paysdelaloire.iufm.fr

Alexandra Wallner est psychologue et prépare actuellement une thèse sur la perception des œuvres d'art par les jeunes enfants en lien avec le type de médiation dont celles-ci sont l'objet. Ses autres thèmes d'étude concernent les processus de promotion de la sécurité routière et l'usage des NTCI en éducation

Adresse : Laboratoire de Psychologie, Université d'Angers, UFR Lettres, Langues et Sciences Humaines, 11, bd Lavoisier, 49045 Angers cedex

Courriel : alexandra.wallner@univ-angers.fr

Annick Weil-Barais est professeur en psychologie cognitive à l'université d'Angers, directrice du laboratoire de psychologie «processus de pensée ». Ses thèmes d'étude concernent l'apprentissage et le développement intellectuel des enfants et des adolescents. Elle s'intéresse actuellement aux transformations des fonctionnements intra et inter-psychiques liées à l'utilisation des NTCI.

Adresse : Laboratoire de Psychologie, Université d'Angers, UFR Lettres, Langues et Sciences Humaines, 11, bd Lavoisier, 49045 Angers cedex

Courriel : annick.weil-barais@univ-angers.fr

\section{Référence de l'article :}

Aurélie LAINÉ, Alexandra WALLNER, Annick WEIL-BARAIS , Evaluation d'un logiciel éducatif dans deux contextes de communication : face à face et synchrone à distance, Revue STICEF, Volume 13, 2006, ISSN : 1764-7223, mis en ligne le 01/02/2007, http://sticef.org

(C) Revue Sciences et Technologies de 1’Information et de la Communication pour l'Éducation et la Formation, 2007 